\title{
Roman domination number of the Cartesian products of paths and cycles
}

\author{
Polona Pavlič \\ Institute of Mathematics, Physics and Mechanics \\ Jadranska 19, 1000 Ljubljana, Slovenia \\ polona.pavlic@imfm.si \\ Janez Žerovnik \\ Faculty of Mechanical Engineering, University of Ljubljana \\ Aškerčeva 6, Ljubljana, Slovenia, and \\ Institute of Mathematics, Physics and Mechanics \\ Jadranska 19, 1000 Ljubljana, Slovenia \\ janez.zerovnik@fs.uni-lj.si
}

Submitted: Nov 16, 2011; Accepted: Aug 1, 2012; Published: Aug 9, 2012

Mathematics Subject Classifications: 05C25, 05C69, 05C85, 68R10

\begin{abstract}
Roman domination is an historically inspired variety of domination in graphs, in which vertices are assigned a value from the set $\{0,1,2\}$ in such a way that every vertex assigned the value 0 is adjacent to a vertex assigned the value 2 . The Roman domination number is the minimum possible sum of all values in such an assignment. Using an algebraic approach we present an $O(C)$-time algorithm for computing the Roman domination numbers of special classes of graphs called polygraphs, which include rotagraphs and fasciagraphs. Using this algorithm we determine formulas for the Roman domination numbers of the Cartesian products of the form $P_{n} \square P_{k}$, $P_{n} \square C_{k}$, for $k \leqslant 8$ and $n \in \mathbb{N}$, and $C_{n} \square P_{k}$ and $C_{n} \square C_{k}$, for $k \leqslant 6$ and $n \in \mathbb{N}$, for paths $P_{n}$ and cycles $C_{n}$. We also find all special graphs called Roman graphs in these families of graphs.
\end{abstract}

\section{Introduction}

The domination number of a graph and its many variations have been extensively studied in the literature along with their algorithmic aspects [24, 23]. It is well known that the problem of computing the domination number of an arbitrary graph is NP-complete [24]. It is therefore worthwhile to construct algorithms for computing domination numbers of 
graphs in various subclasses of graphs. Among them, grids (i.e. Cartesian products of paths) are widely studied because they represent interconnection models of multiprocessors in VLSI systems. The domination numbers of the Cartesian products, for several fixed values of $k$, were computed for $P_{n} \square P_{k}$ in $[1,8,11,19,20]$, for $C_{n} \square C_{k}$ in $[14,35,52]$ and for $P_{n} \square C_{k}$ in [42]. A general $\mathrm{O}(\log n)$ algorithm based on path algebra in [36], can be used to compute the domination number of $P_{n} \square P_{k}$, for any fixed $k$. This algorithm can also be used to compute distance based invariants [32] and domination numbers [53] in polygraphs in constant time, that is, the algorithm can find closed formulas for arbitrary values of $n$. The existence of an algorithm that can provide closed formulas for the domination numbers of all grid graphs $\left(P_{n} \square P_{k}\right)$ has been observed or claimed in [17, 40].

An interesting variety of domination that is popular because of its historical significance is called Roman domination [43, 48]. In the 4th century Emperor Constantine, in order to defend the Roman Empire, decreed that two types of armies should be placed in cities in such a way that the entire Empire could be secured. The first type of army was highly trained and mobile, and could move from city to city to defend against any attack. The second type of army was a local militia that was permanently stationed at a given city. The Emperor decreed that no mobile army could ever leave a city to defend another if in doing so it left the originating city undefended. Thus, two armies were stationed at some cities, only a local militia at others, and other cities had no army.

While the problem is still of interest in military operations research [2], it also has applications in cases where a time-critical service is to be provided with some backup. For example, a fire station should never send all emergency vehicles to answer a call.

In Roman domination, every vertex is assigned a value from the set $\{0,1,2\}$ in such a way that every vertex assigned the value 0 is adjacent to at least one vertex assigned the value 2. The Roman domination number equals the minimum sum of all values in such an assignment. A formal definition of the Roman domination number of a graph, first given in [9], is presented in Section 2.

Since the problem of computing the Roman domination number of an arbitrary graph is NP-complete [12], it is worthwhile to compute the Roman domination number of various classes of graphs $[9,18,25,39,38,46,47]$. A Vizing-like conjecture for Roman domination [49], some properties of minimum Roman dominating functions [7, 15, 29, 41, 50] and algorithmic aspects of Roman domination [16] have also been studied. One of the open problems in [9] was that of computing the Roman domination numbers of arbitrary grid graphs. The Roman domination numbers of grid graphs $P_{n} \square P_{k}$ have been determined for $k \leqslant 4$ and $n \in \mathbb{N}$ in $[9,12]$. A linear-time algorithm for computing the Roman domination number of $P_{n} \square P_{k}$, for any fixed $k$, is given in [12].

In this paper we use the path algebra approach [36] to design an $O(\log n)$ algorithm for computing the Roman domination numbers of arbitrary grid graphs, and show how it can be converted to a constant time algorithm that provides closed formulas for these numbers. More precisely, the time complexity of this algorithm is independent of $n$ and has superpolynomial time complexity in terms of $k$. We use the algorithm to find formulas for Roman domination numbers of $P_{n} \square P_{k}$ and $P_{n} \square C_{k}$ for $k \leqslant 8$ and $n \in \mathbb{N}$, and for $C_{n} \square P_{k}$ and $C_{n} \square C_{k}$ for $k \leqslant 6$ and $n \in \mathbb{N}$. 
In Section 2 we present the concept of polygraphs, which has been widely used in chemical graph theory and elsewhere $[4,21,31,33]$. In Section 3 we summarize a general framework for solving different problems in the classes of polygraphs, called rotagraphs and faciagraphs, that is presented in [36]. Section 4 contains the algorithm for computing the Roman domination numbers of rotagraphs and faciagraphs. Section 5 summarizes the results obtained by this algorithm, and Section 6 presents a listing of all known Roman graphs in the classes of rotagraphs and faciagraphs studied. We conclude with a couple of remarks and open problems on the topic in Section 7.

\section{Preliminaries}

We consider finite undirected and directed graphs. The term graph denotes an undirected graph, while the term digraph denotes a directed graph. An edge between two vertices $u$ and $v$ in a graph is denoted $u v$, while an arc from a vertex $u$ to a vertex $v$ in digraph is denoted $(u, v)$. Let $P_{n}$ denote a path on $n$ vertices and $C_{n}$ denote a cycle on $n$ vertices.

In a graph $G=(V, E)$, a set $D \subseteq V$ is a dominating set if every vertex in $V \backslash D$ is adjacent to a vertex in $D$. The domination number $\gamma(G)$ equals the minimum cardinality of a dominating set in $G$. A dominating set of cardinality $\gamma(G)$ is called a $\gamma$-set.

Roman domination was formally defined in [9] as follows: For a graph $G=(V, E)$, let $f: V \longrightarrow\{0,1,2\}$ and let $\left(V_{0}, V_{1}, V_{2}\right)$ be the ordered partition of $V$ induced by $f$, where $V_{i}=\{v \in V(G) \mid f(v)=i\}$. Let $\left|V_{i}\right|=n_{i}$ for $i=0,1,2$. Note that there exists a 1-1 correspondence between the functions $f: V \longrightarrow\{0,1,2\}$ and ordered partitions $\left(V_{0}, V_{1}, V_{2}\right)$ of $V$. Thus, we will write $f=\left(V_{0}, V_{1}, V_{2}\right)$. A function $f=\left(V_{0}, V_{1}, V_{2}\right)$ is a Roman dominating function (RDF) if every vertex in $V_{0}$ is adjacent to a vertex in $V_{2}$. The weight of $f$ is defined as:

$$
w(f)=\sum_{v \in V} f(v)=n_{1}+2 n_{2}
$$

The Roman domination number, $\gamma_{R}(G)$, equals the minimum weight of an RDF of $G$. We will also say that a function $f=\left(V_{0}, V_{1}, V_{2}\right)$ is a $\gamma_{R}$-function, if it is an RDF and $w(f)=$ $\gamma_{R}(G)$. Obviously, $\gamma(G) \leqslant \gamma_{R}(G) \leqslant 2 \gamma(G)$. The only graphs that satisfy $\gamma_{R}(G)=\gamma(G)$ are edgeless graphs. A graph is called a Roman graph if $\gamma_{R}(G)=2 \gamma(G)$. Finding classes of Roman graphs was one of the open problems posed in [9]. For instance, among paths, graphs $P_{3 k}$ and $P_{3 k+2}$ are Roman graphs. Paths $P_{3 k+1}$ are examples of graphs which are not Roman graphs: $\gamma_{R}\left(P_{3 k+1}\right)=2 k+1<2 \gamma\left(P_{3 k+1}\right)=2 k+2$.

The difference between $\gamma_{R}$ and $2 \gamma$ can be arbitrarily large. Consider, for example, the family of subdivided stars $S\left(K_{1, n}\right)$, which are obtained from the family of stars $K_{1, n}$ by subdividing every edge. It can be seen that $\gamma_{R}\left(S\left(K_{1, n}\right)\right)=2+n$, while $2 \gamma\left(S\left(K_{1, n}\right)\right)=2 n$. The construction of a $\gamma$-set and $\gamma_{R}$-function for $S\left(K_{1,5}\right)$ is given in Figure 1, where the full circs in the subdivided star on the left are elements of a $\gamma$-set, while the full circs in the subdivided star on the right are elements of $V_{1}$ and the double circ vertex in the center is the only element of $V_{2}$ in the $\gamma_{R}$-function. 

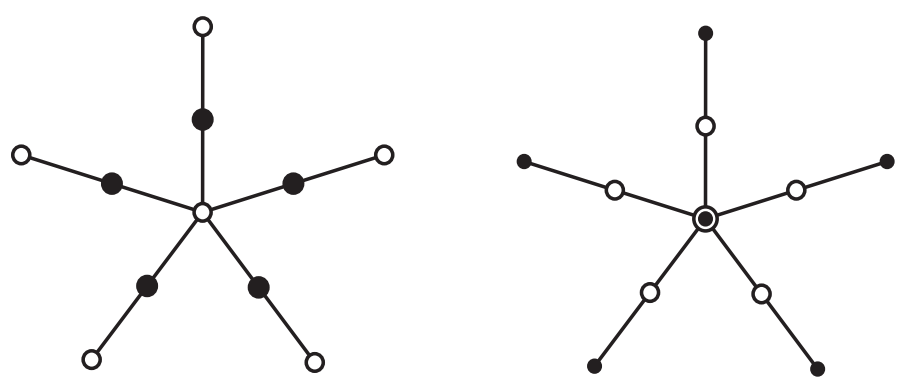

Figure 1: A minimum dominating set and a $\gamma_{R}$-function of $S\left(K_{1,5}\right)$.

The Cartesian product of graphs $G$ and $H$, denoted $G \square H$, is a graph with a vertex set $V(G) \times V(H)$ and $(g, h)\left(g^{\prime}, h^{\prime}\right) \in E(G \square H)$ if $g=g^{\prime}$ and $h h^{\prime} \in E(H)$ or $g g^{\prime} \in E(G)$ and $h=h^{\prime}$. Cartesian product graphs include the grid graphs, which are products of paths $P_{n} \square P_{k}$, and tori, which are products of cycles $C_{n} \square C_{k}$.

Let $G_{1}, \ldots, G_{n}$ be arbitrary mutually disjoint graphs and $X_{1}, \ldots, X_{n}$ a sequence of sets of edges such that an edge of $X_{i}$ joins a vertex of $V\left(G_{i}\right)$ with a vertex of $V\left(G_{i+1}\right)$ $\left(X_{i} \subseteq V\left(G_{i}\right) \times V\left(G_{i+1}\right)\right.$ for $\left.i=1, \ldots, n\right)$. For convenience we also set $G_{n+1}=G_{1}$. A polygraph $\Omega_{n}=\Omega_{n}\left(G_{1}, \ldots G_{n} ; X_{1}, \ldots X_{n}\right)$ over monographs $G_{1}, \ldots, G_{n}$ is defined in the following way:

$$
\begin{gathered}
V\left(\Omega_{n}\right)=V\left(G_{1}\right) \cup \ldots \cup V\left(G_{n}\right), \\
E\left(\Omega_{n}\right)=E\left(G_{1}\right) \cup X_{1} \cup \ldots \cup E\left(G_{n}\right) \cup X_{n} .
\end{gathered}
$$

For a polygraph $\Omega_{n}$ and for $i=1, \ldots, n$ we also define

$$
\begin{aligned}
& D_{i}=\left\{u \in V\left(G_{i}\right) \mid \exists v \in G_{i+1}: u v \in X_{i}\right\}, \\
& R_{i}=\left\{u \in V\left(G_{i+1}\right) \mid \exists v \in G_{i}: u v \in X_{i}\right\} .
\end{aligned}
$$

In general, $R_{i} \cap D_{i+1}$ does not have to be empty. If all graphs $G_{i}$ are isomorphic to a fixed graph $G$ (i.e. there exists an isomorphism $\varphi_{i}: V\left(G_{i}\right) \longrightarrow V(G)$ for $i=1, \ldots, n+1$, and $\left.\varphi_{n+1}=\varphi_{1}\right)$ and all sets $X_{i}$ are equal to a fixed set $X \subseteq V(G) \times V(G)((u, v) \in X \Longleftrightarrow$ $\left(\varphi_{i}^{-1}(u), \varphi_{i+1}^{-1}(v)\right) \in X_{i}$ for all $\left.i\right)$, we call such a graph rotagraph, $\omega_{n}(G ; X)$. A rotagraph without edges between the first and the last copy of $G$ is a fasciagraph, $\psi_{n}(G ; X)$. More precisely, in a fasciagraph, $X_{n}=\emptyset$ and $X_{1}=X, \ldots, X_{n-1}=X$. In a rotagraph as well as in a fasciagraph, all sets $D_{i}$ and $R_{i}$ are equal to fixed sets $D$ and $R$, respectively $\left(D_{i}=\varphi_{i}^{-1}(D)\right.$ and $\left.R_{i}=\varphi_{i+1}^{-1}(R)\right)$. Of course, in the case of fasciagraphs, $D_{n}=\emptyset$ and $R_{n}=\emptyset$. Observe that the Cartesian product of paths $P_{n}$ and $P_{k}, P_{n} \square P_{k}$, is an example of a fasciagraph and that the Cartesian product of cycles $C_{n}$ and $C_{k}, C_{n} \square C_{k}$, is an example of a rotagraph. A graph $P_{n} \square C_{k}$ can be treated either as a fasciagraph or as a rotagraph.

\section{Path algebras and the algorithm}

Let us now summarize a general framework for solving different problems on the class of fasciagraphs and rotagraphs, which was proposed in [36] and also used in [53]: 
A semiring $\mathcal{P}=\left(P, \oplus, \circ, e^{\oplus}, e^{\circ}\right)$ is a set $P$ on which two binary operations, $\oplus$ and $\circ$ are defined such that:

1. $(P, \oplus)$ is a commutative monoid with $e^{\oplus}$ as a unit;

2. $(P, \circ)$ is a monoid with $e^{\circ}$ as a unit;

3. $\circ$ is left- and right-distributive over $\oplus$;

4. $\forall x \in P, x \circ e^{\oplus}=e^{\oplus}=e^{\oplus} \circ x$.

An idempotent semiring is called a path algebra. It is easy to see that a semiring is a path algebra if and only if $e^{\circ} \oplus e^{\circ}=e^{\circ}$ holds for $e^{\circ}$, the unit of the monoid $(P, \circ)$. An important example of a path algebra for our work is $\mathcal{P}_{1}=\left(\mathbb{N}_{0} \cup\{\infty\}, \min ,+, \infty, 0\right)$. Here $\mathbb{N}_{0}$ denotes the set of nonnegative integers and $\mathbb{N}$ the set of positive integers. For more examples of path algebras we refer to [6].

Let $\mathcal{P}=\left(P, \oplus, \circ, e^{\oplus}, e^{\circ}\right)$ be a path algebra and let $\mathcal{M}_{n}(\mathcal{P})$ be the set of all $n \times n$ matrices over $P$. Let $A, B \in \mathcal{M}_{n}(\mathcal{P})$ and define operations $\oplus$ and $\circ$ in the usual way:

$$
\begin{aligned}
(A \oplus B)_{i j} & =A_{i j} \oplus B_{i j}, \\
(A \circ B)_{i j} & =\bigoplus_{k=1}^{n} A_{i k} \circ B_{k j} .
\end{aligned}
$$

$\mathcal{M}_{n}(\mathcal{P})$ equipped with the above defined operations is a path algebra with the zero and the unit matrix as units of the semiring. In our example $\mathcal{P}_{1}=\left(\mathbb{N}_{0} \cup\{\infty\}\right.$, min, $\left.+, \infty, 0\right)$, all elements of the zero matrix are $\infty$, the unit of the monoid $(P, \min )$, and the unit matrix is a diagonal matrix with diagonal elements equal to $e^{\circ}=0$ and all other elements equal to $e^{\oplus}=\infty$.

Let $\mathcal{P}$ be a path algebra and let $G$ be a labeled digraph, that is a digraph together with a labeling function $\ell$ which assigns to every arc of $G$ an element of $P$. Let $V(G)=$ $\left\{v_{1}, v_{2}, \ldots, v_{n}\right\}$. The labeling $\ell$ of $G$ can be extended to walks in the following way: For a walk $Q=\left(v_{i_{0}}, v_{i_{1}}\right)\left(v_{i_{1}}, v_{i_{2}}\right) \ldots\left(v_{i_{k-1}}, v_{i_{k}}\right)$ of $G$ let

$$
\ell(Q)=\ell\left(v_{i_{0}}, v_{i_{1}}\right) \circ \ell\left(v_{i_{1}}, v_{i_{2}}\right) \circ \ldots \circ \ell\left(v_{i_{k-1}}, v_{i_{k}}\right) .
$$

Let $S_{i j}^{k}$ be the set of all walks of order $k$ from $v_{i}$ to $v_{j}$ in $G$ and let $A(G)$ be the matrix defined by:

$$
A(G)_{i j}= \begin{cases}\ell\left(v_{i}, v_{j}\right) ; & \text { if }\left(v_{i}, v_{j}\right) \text { is an arc of } G \\ e^{\oplus} ; & \text { otherwise. }\end{cases}
$$

It is well-known (see for example [6]) that

$$
\left(A(G)^{k}\right)_{i j}=\bigoplus_{Q \in S_{i j}^{k}} \ell(Q) .
$$

Let $\omega_{n}(G ; X)$ be a rotagraph and $\psi_{n}(G ; X)$ a fasciagraph. Set $U=D_{i} \cup R_{i}=D \sqcup R$ and let $N=2^{|U|}$. Define a labeled digraph $\mathcal{G}=\mathcal{G}(G ; X)$ as follows: The vertex set 
of $\mathcal{G}$ is formed by the subsets of $U$ which will be denoted $V_{i}$. An arc joins a subset $V_{i}$ with a subset $V_{j}$ if $V_{i}$ is not in a "conflict" with $V_{j}$. Here a conflict of $V_{i}$ with $V_{j}$ means that using $V_{i}$ and $V_{j}$ as a part of a solution in consecutive copies of $G$ would violate the problem assumption. For instance, if we look for a domination number of a graph, such a conflict would be a nonempty intersection between sets $V_{i}$ and $V_{j}$, or if we look for an independence number of a graph, such a conflict would be an edge between sets $V_{i}$ and $V_{j}$. Let finally $\ell: E(\mathcal{G}) \longrightarrow P$ be a labeling of $\mathcal{G}$ where $\mathcal{P}$ is a path algebra on the set $P$. The general scheme for the algorithm that solves different problems on polygraphs as proposed in [36] is:

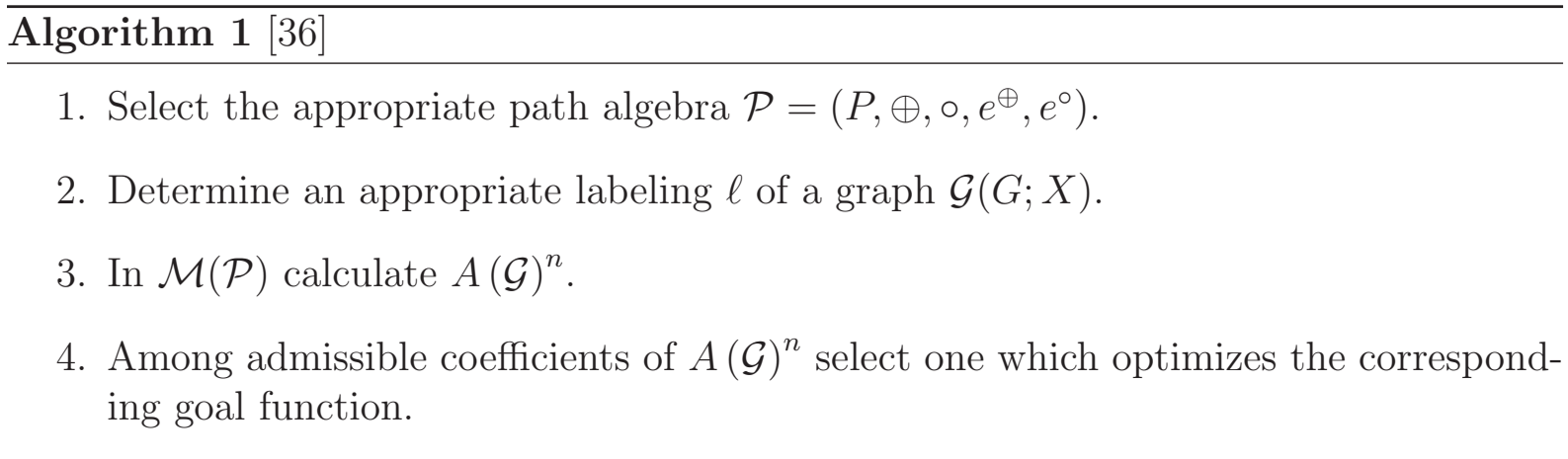

It is well known that, in general, Step 3 of the algorithm can be implemented to run in $O(\log n)$-time [44]. However, computing the powers of $A(\mathcal{G})^{n}=A_{n}$ in $O(C)$-time is possible using special structure of the matrices in some cases, including the distance based invariants [32], the domination numbers [53], and others [31, 33, 54]. Here we prove that $A(\mathcal{G})^{n}=A_{n}$ can be computed in $O(C)$-time for the Roman domination number (see Section 4).

\section{The Roman domination number of fasciagraphs and rotagraphs}

Let $\omega_{n}(G ; X)$ be a rotagraph and $\psi_{n}(G ; X)$ a fasciagraph as defined above. Set $U=$ $D_{i} \cup R_{i}=D \sqcup R$. Keep in mind that $D_{i} \subseteq G_{i}$ and $R_{i} \subseteq G_{i+1}$, but since $R_{i}=R$ and $D_{i}=D$ for all $i$, we can write $U=D_{i} \cup R_{i}=D \sqcup R$. A labeled digraph $\mathcal{G}=\mathcal{G}(G ; X)$ is a graph with a vertex set:

$$
V(\mathcal{G})=\left\{\left(V_{i}, W_{i}\right) \mid V_{i}, W_{i} \subseteq U, V_{i} \cap W_{i}=\emptyset\right\}
$$

For convenience we sometimes refer to a vertex of $\mathcal{G}$ shortly by $v_{i}=\left(V_{i}, W_{i}\right)$. In particular, $v_{0}=\left(V_{0}, W_{0}\right)$ stands for $(\emptyset, \emptyset)$.

Let $v_{i}, v_{j} \in V(\mathcal{G})$ and consider for a moment $\psi_{3}(G ; X)$. Let $V_{i} \cup W_{i} \subseteq D_{1} \cup R_{1}$ and $V_{j} \cup W_{j} \subseteq D_{2} \cup R_{2}$. Let $\gamma_{R_{i, j}}(G ; X)$ be the weight of a $\gamma_{R^{-}}$function of a graph $G_{2} \backslash\left(\left(\left(V_{i} \cup W_{i}\right) \cap R_{1}\right) \cup\left(D_{2} \cap\left(V_{j} \cup W_{j}\right)\right)\right)$, such that $V_{i} \cup V_{j} \subseteq V^{\prime}$ and $W_{i} \cup W_{j} \subseteq W^{\prime}$, 


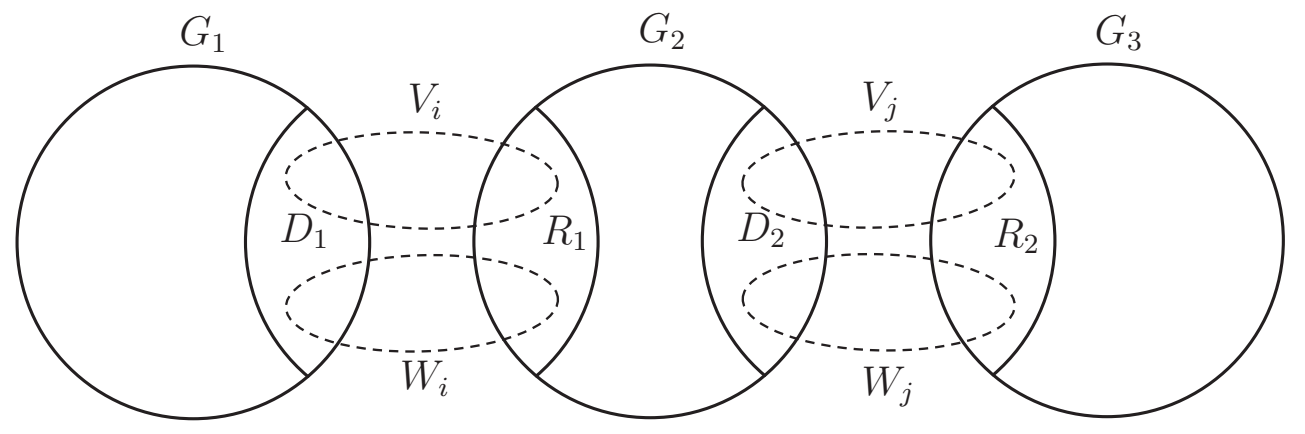

Figure $2: \psi_{3}(G ; X)$ with the above notation.

where $\left(V\left(G_{2}\right) \backslash\left(V^{\prime} \cup W^{\prime}\right), V^{\prime}, W^{\prime}\right)$ is an RDF of a graph $G_{2}$. For consistency, we introduce an arc between vertices $v_{i}$ and $v_{j}$ only if $R \cap V_{i} \cap W_{j} \cap D=\emptyset$ and $R \cap W_{i} \cap V_{j} \cap D=\emptyset$. Set

$$
\begin{aligned}
\ell\left(v_{i}, v_{j}\right) & =\left|R \cap V_{i}\right|+2\left|R \cap W_{i}\right|+\left|V_{j} \cap D\right|+2\left|W_{j} \cap D\right|- \\
& -\left|R \cap V_{i} \cap V_{j} \cap D\right|-2\left|R \cap W_{i} \cap W_{j} \cap D\right|+\gamma_{R_{i, j}}(G ; X) .
\end{aligned}
$$

Then we have an algorithm which computes the Roman domination number of rotagraphs and fasciagraphs:

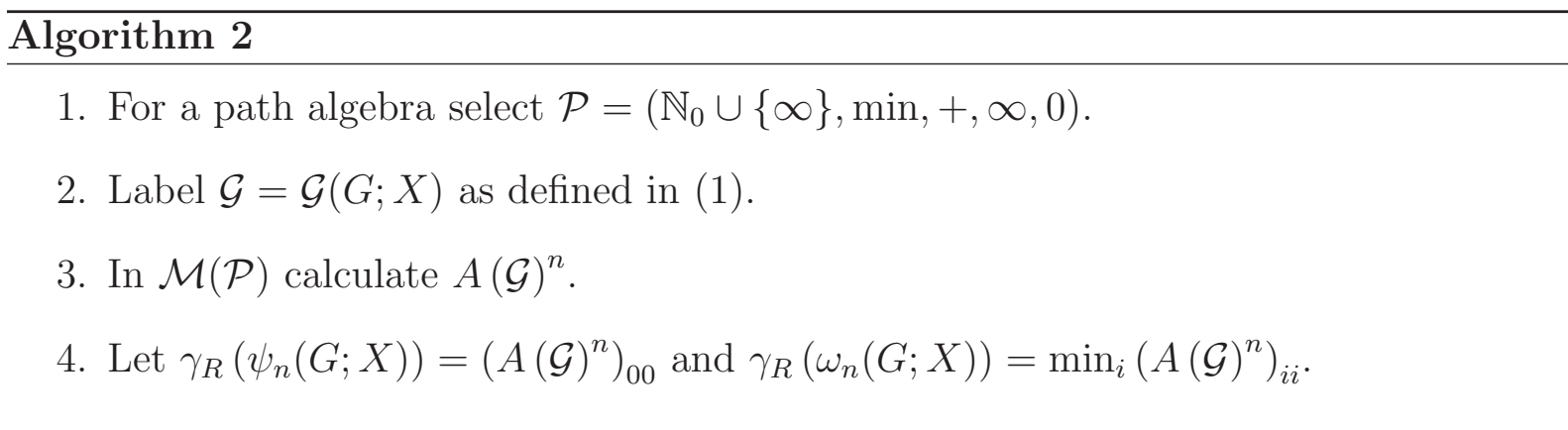

Theorem 4.1 The Algorithm 2 correctly computes the Roman domination number of rotagraphs and fasciagraphs:

$$
\begin{gathered}
\gamma_{R}\left(\psi_{n}(G ; X)\right)=\left(A(\mathcal{G})^{n}\right)_{00} \\
\gamma_{R}\left(\omega_{n}(G ; X)\right)=\min _{i}\left(A(\mathcal{G})^{n}\right)_{i i}
\end{gathered}
$$

in $O(\log n)$-time.

Proof. Let $G_{1}$ and $G_{2}$ be arbitrary graphs, $X_{1}$ a set of edges between vertices of $G_{1}$ and $G_{2}$ and let $\Omega_{2}\left(G_{1}, G_{2} ; X_{1}, \emptyset\right)$ be a polygraph. Let also $\mathcal{P}=\left(\mathbb{N}_{0} \cup\{\infty\}\right.$, min, $\left.+, \infty, 0\right)$ 
be a path algebra and let $\mathcal{G}^{\prime}$ be a labeled digraph for $\Omega_{2}$ defined as above. Then, by the definition of the labeling, we have

$$
\begin{aligned}
\gamma_{R}\left(\Omega_{2}\left(G_{1}, G_{2} ; X_{1}, \emptyset\right)\right) & =\left[A\left(G_{1}\right)+A\left(G_{2}\right)\right]_{00} \\
& =\left[\min _{v_{k} \in V(\mathcal{G})}\left\{\ell\left(0, v_{k}\right)+\ell\left(v_{k}, 0\right)\right\}\right]_{00} .
\end{aligned}
$$

Let $G_{1}=G, X_{1}=X$ and $G_{2}=\psi_{n-1}(G ; X)$. Then (2) follows by induction.

For (3), similarly, consider $\Omega_{2}\left(G_{1}, G_{2} ; X_{1}, X_{2}\right)$ and let $G_{1}=G, X_{1}=X_{2}=X$ and $G_{2}=\psi_{n-1}(G ; X)$.

The time complexity of the algorithm was already discussed for a general case in Section 3.

As mentioned before we prove that calculating the powers of matrices $A(\mathcal{G}), A(\mathcal{G})^{n}=$ $A_{n}$ (and therefore implementing the algorithm) is possible in $O(C)$-time based on the following lemma:

Lemma 4.2 Let $k=|V(\mathcal{G}(G ; X))|$ and $K=|V(G)|$. Then there is an index $q \leqslant(4 K+$ $2)^{k^{2}}$ such that $A_{q}=A_{p}+C$ for some index $p<q$ and some constant matrix $C$. Let $P=q-p$. Then for every $r \geqslant p$ and every $s \geqslant 0$ we have

$$
A_{r+s P}=A_{r}+s C \text {. }
$$

Proof. First observe that for any $l \geqslant 1$, the difference between any pair of entries of $A_{l}$, both different from $\infty$, is bounded by $4 K$. Assume $\left(A_{l}\right)_{i j} \neq \infty$. Then

$$
\begin{aligned}
\left(A_{l}\right)_{i j} & =\gamma_{R}\left(\left(V\left(G_{1}\right) \backslash\left(V_{i} \cup W_{i}\right)\right) \cup V\left(G_{2}\right) \cup \ldots \cup V\left(G_{l-1}\right) \cup\left(V\left(G_{l}\right) \backslash\left(V_{j} \cup W_{j}\right)\right)\right) \\
& \leqslant \gamma_{R}\left(\psi_{l}(G ; X)\right) .
\end{aligned}
$$

Since $V_{i} \cap W_{i}=\emptyset$ and $V_{j} \cap W_{j}=\emptyset$ it follows that

$$
\left|R \cap V_{i}\right|+2\left|R \cap W_{i}\right|+\left|V_{j} \cap D\right|+2\left|W_{j} \cap D\right| \leqslant 4|V(G)| .
$$

According to (1) we have

$$
\begin{aligned}
\ell\left(v_{i}, v_{j}\right) & \leqslant 4|V(G)|+\gamma_{R_{i, j}}=4|V(G)|+\left(A_{l}\right)_{i j} \\
\quad\left(A_{l}\right)_{i j} & \geqslant \ell\left(v_{i}, v_{j}\right)-4|V(G)| \geqslant \gamma_{R}\left(\psi_{l}(G ; X)\right)-4|V(G)| .
\end{aligned}
$$

Therefore

$$
\gamma_{R}\left(\psi_{l}(G ; X)\right)-4|V(G)| \leqslant\left(A_{l}\right)_{i j} \leqslant \gamma_{R}\left(\psi_{l}(G ; X)\right) .
$$

For $l \geqslant 1$, let $K_{l}=\min \left\{\left(A_{l}\right)_{i j}\right\}$ and let $A_{l}^{\prime}=A_{l}-\left(K_{l}\right) J$, where $J$ is the matrix with all entries equal to 0 (recall that we are still in the path algebra $\mathcal{P}=\left(\mathbb{N}_{0} \cup\{\infty\}\right.$, min, $\left.+, \infty, 0\right)$ ). Since the difference between any two elements of $A_{l}$, different from $\infty$, cannot be greater than $4 K$, the entries of $A_{l}^{\prime}$ can only have values $0,1, \ldots, 4 K, \infty$. Hence there are indices $p<q \leqslant(4 K+2)^{k^{2}}$ such that $A_{p}^{\prime}=A_{q}^{\prime}$. This proves the first part of the lemma. 
The equality $A_{r+s P}=A_{r}+s C$ follows from the fact that for arbitrary matrices $D, E$ and a constant matrix $C$ we have $(D+C) \circ E=D \circ E+C$, where + is the ordinary matrix addition, i.e. $(A+B)_{i j}=A_{i j}+B_{i j}$ for all $i, j$ :

$$
\begin{aligned}
((D+C) \circ E)_{i j} & =\min _{k}\left\{\left((D)_{i k}+C\right)+(E)_{k j}\right\}=\min _{k}\left\{(D)_{i k}+(E)_{k j}\right\}+C \\
(D \circ E+C)_{i j} & =\min _{k}\left\{(D)_{i k}+(E)_{k j}\right\}+(C)_{i j}=\min _{k}\left\{(D)_{i k}+(E)_{k j}\right\}+C .
\end{aligned}
$$

Therefore, let $A_{q}=A_{p}+C$ for some index $p<q$ and some constant matrix $C=[c]_{i j}$. Then

$$
\begin{aligned}
A_{q+1} & =\left(A_{p}+C\right) \circ A \\
& =\left(A_{p} \circ A\right)+C \\
& =A_{p+1}+C .
\end{aligned}
$$

Let $P=q-p$ and $r \geqslant p$. Then also

$$
\begin{aligned}
A_{r+P} & =A_{r+q-p}=A_{q} \circ A_{r-p} \\
& =\left(A_{p}+C\right) \circ A_{r-p}=A_{p+r-p}+C \\
& =A_{r}+C,
\end{aligned}
$$

and by induction on $s$ we have

$$
\begin{aligned}
A_{r+s P} & =A_{r+P+(s-1) P}=\left(A_{r}+C\right) \circ A_{(s-1) P}=A_{r+(s-1) P}+C \\
& =A_{r}+(s-1) C+C=A_{r}+s C
\end{aligned}
$$

for every $s \geqslant 0$.

Remark 4.3 Consider the Roman domination number of a fasciagraph $P_{n} \square P_{k}$, where $k$ is a fixed number. Then $q<(4 k+2)^{2^{3 k}}$. In Table 1 we give the upper bounds for $q$ for the investigated grids. Observe that already in the cases of very small monographs, enormously large $q$ are obtained. That is why the second part of Lemma 4.2 is useful for practical purposes - once a period is detected, it cannot change. As we implemented the improved algorithm, the period was always found much sooner. Exact values are presented in the third column of Table 1.

Hence, Lemma 4.2 says that if we assume the size of $G$ is a given constant (and $n$ is a variable), then the algorithm will run in constant time. But it is important to emphasize that the algorithm is useful for practical purposes only if the number of vertices of the monograph $G$ is relatively small, since the time complexity is in general exponential in the number of vertices of the monograph $G$. 
Table 1: The upper bound for $q$ and actual values computed by the algorithm.

\begin{tabular}{ccc}
\hline$P_{n} \square P_{k}$ & $\begin{array}{c}\text { the upper bound for } \\
\text { from Lemma } 4.2\end{array}$ & $\begin{array}{c}\text { value computed by } \\
\text { the algorithm }\end{array}$ \\
\hline$k=2$ & $10^{64}$ & 3 \\
\hline$k=3$ & $14^{512} \approx 6 \cdot 10^{586}$ & 6 \\
\hline$k=4$ & $18^{4096} \approx 4 \cdot 10^{5141}$ & 9 \\
\hline$k=5$ & $22^{32768}$ & 11 \\
\hline$k=6$ & $26^{262144}$ & 9 \\
\hline$k=7$ & $30^{2097152}$ & 16 \\
\hline$k=8$ & $34^{16777216}$ & 11 \\
\hline
\end{tabular}

\section{Cartesian products of paths and cycles}

Our aim is to calculate the Roman domination number for graphs $P_{n} \square P_{k}, P_{n} \square C_{k}, C_{n} \square P_{k}$ and $C_{n} \square C_{k}$ for some fixed values of $k$. These graphs are isomorphic to special classes of fasciagraphs and rotagraphs (i.e. fasciagraphs and rotagraphs where $G=P_{k}$ or $G=$ $C_{k}$ and where $X$ is a matching between two copies of $G$ ). Therefore, calculating exact formulas for their Roman domination number is possible because of the Algorithm 2 together with Lemma 4.2. But with increasing $k$ matrices $A(G)^{n}$ become bigger and bigger. Therefore we had to improve straightforward implementation of the algorithm. Instead of calculating all elements of the powers of matrices $A(G)^{n}$, we calculated only those rows which are important for the result. Then we checked the difference of the new row against the previously stored rows until a constant difference was detected. This yields a correct result because of the next lemma, adaptation of a lemma from [54].

Lemma 5.1 Assume that the $j$-th row of $A_{n+P}$ and $A_{n}$ differ for a constant, $a_{j i}^{(n+P)}=$ $a_{j i}^{(n)}+C$ for all $i$. Then $\min _{i} a_{j i}^{(n+P)}=\min _{i} a_{j i}^{(n)}+C$.

Proof. Let $a_{j l}^{(n+P)}=\min _{i} a_{j i}^{(n+P)}$ and assume that there exists $t \neq l$ such that $a_{j l}^{(n)}>a_{j t}^{(n)}$. It follows that

$$
a_{j t}^{(n+P)}=a_{j t}^{(n)}+C<a_{j l}^{(n)}+C=a_{j l}^{(n+P)},
$$

which contradicts the minimality of $a_{j l}^{(n+P)}$.

Obviously, such an improvement is crucial in the case of fasciagraphs: recall that $\gamma_{R}\left(\psi_{n}(G ; X)\right)=\left(A(\mathcal{G})^{n}\right)_{00}$. For computing 00-th element of $A_{n}$ we only need the first rows of the matrices $A_{j}$ for $2 \leqslant j \leqslant n-1$ :

$$
A_{n_{0 j}}=\min _{i}\left\{A_{n-1_{0 i}}+A_{i j}\right\} \text {. }
$$


This is not the case for rotagraphs because $\gamma_{R}\left(\omega_{n}(G ; X)\right)=\min _{i}\left(A(\mathcal{G})^{n}\right)_{i i}$.

By implementation of the improved algorithm we got formulas presented in the following subsections. For each case also constructions of $\gamma_{R}$-functions are presented. In every figure that follows we only emphasized vertices of $V_{1}$ and $V_{2}$ of a $\gamma_{R}$-function of a depicted graph in a way that a single full circ represents a vertex of $V_{1}$ and a double circ represents a vertex of $V_{2}$.

\section{$5.1 \gamma_{R}\left(P_{n} \square P_{k}\right)$ for $k \in\{5,6,7,8\}$}

The Roman domination number of grid graphs was studied in $[9,12]$ and the following results were established:

$$
\begin{aligned}
\gamma_{R}\left(P_{n}\right) & =\gamma_{R}\left(C_{n}\right)=\left\lceil\frac{2 n}{3}\right\rceil \\
\gamma_{R}\left(P_{n} \square P_{2}\right) & =n+1 \\
\gamma_{R}\left(P_{n} \square P_{3}\right) & = \begin{cases}\left\lfloor\frac{3 n}{2}\right\rfloor+2 ; & \text { if } n \in\{4 k+3 \mid k \in \mathbb{N} \cup\{0\}\} \\
\left\lfloor\frac{3 n}{2}\right\rfloor+1 ; & \text { otherwise }\end{cases} \\
\gamma_{R}\left(P_{n} \square P_{4}\right) & = \begin{cases}2 n+1 ; & \text { if } n \in\{1,2,3,5,6\} \\
2 n ; & \text { otherwise }\end{cases}
\end{aligned}
$$

No formulas were given for $k>4$. However, in [12] the author also proposed an algorithm for computing $\gamma_{R}\left(P_{n} \square P_{k}\right)$ for any fixed value of $k$ in $O(n)$-time. By implementing the improved Algorithm 2 as already discussed above, we obtained formulas given below. We also looked for the $\gamma_{R}$-functions of graphs $P_{n} \square P_{k}$ for the investigated $k$ and all $n \in \mathbb{N}$. They are depicted in Figures 3 to 6.

$$
\begin{aligned}
& \gamma_{R}\left(P_{n} \square P_{5}\right)= \begin{cases}8 ; & \text { if } n=3 \\
\left\lfloor\frac{12 n}{5}\right\rfloor+2 ; & \text { otherwise }\end{cases} \\
& \gamma_{R}\left(P_{n} \square P_{6}\right)= \begin{cases}\left\lfloor\frac{14 n}{5}\right\rfloor+2 ; & \text { if } n<5 \text { or } n \in\{5 k, 5 k+3,5 k+4 \mid k \in \mathbb{N}\} \\
\left\lfloor\frac{14 n}{5}\right\rfloor+3 ; & \text { otherwise }\end{cases} \\
& \gamma_{R}\left(P_{n} \square P_{7}\right)= \begin{cases}\left\lfloor\frac{16 n}{5}\right\rfloor+2 ; & \text { if } n \in\{1,2,4,7,5 k \mid k \in \mathbb{N}\} \\
\left\lfloor\frac{16 n}{5}\right\rfloor+3 ; & \text { otherwise }\end{cases} \\
& \gamma_{R}\left(P_{n} \square P_{8}\right)= \begin{cases}9 ; & \text { if } n=2 \\
16 ; & \text { if } n=4 \\
\left\lfloor\frac{18 n}{5}\right\rfloor+4 ; & \text { if } n \in\{5 k+3 \mid k \in \mathbb{N}\} \\
\left\lfloor\frac{18 n}{5}\right\rfloor+3 ; & \text { otherwise }\end{cases}
\end{aligned}
$$

The Roman domination numbers for small grids are presented in Table 2. 
Table 2: Roman domination number of some $P_{n} \square P_{k}$.

\begin{tabular}{cccccccccccccccc}
\hline$k \backslash n$ & 1 & 2 & 3 & 4 & 5 & 6 & 7 & 8 & 9 & 10 & 11 & 12 & 13 & 14 & 15 \\
\hline 1 & 1 & 2 & 2 & 3 & 4 & 4 & 5 & 6 & 6 & 7 & 8 & 8 & 9 & 10 & 10 \\
2 & & 3 & 4 & 5 & 6 & 7 & 8 & 9 & 10 & 11 & 12 & 13 & 14 & 15 & 16 \\
3 & & & 6 & 7 & 8 & 10 & 12 & 13 & 14 & 16 & 18 & 19 & 20 & 22 & 24 \\
4 & & & & 8 & 11 & 13 & 14 & 16 & 18 & 20 & 22 & 24 & 26 & 28 & 30 \\
5 & & & & & 14 & 16 & 18 & 21 & 23 & 26 & 28 & 30 & 33 & 35 & 38 \\
6 & & & & & & 19 & 22 & 24 & 27 & 30 & 33 & 36 & 38 & 41 & 44 \\
7 & & & & & & & 24 & 28 & 31 & 34 & 38 & 41 & 44 & 47 & 50 \\
8 & & & & & & & & 32 & 35 & 39 & 42 & 46 & 50 & 53 & 57 \\
\hline
\end{tabular}

\section{$5.2 \gamma_{R}\left(P_{n} \square C_{k}\right)$ for $k \in\{3,4,5,6,7,8\}$}

In the literature we found no results on the Roman domination numbers of graphs $P_{n} \square C_{k}$. Our formulas are given below and constructions for each case are depicted in Figures 7 to 12 .

$$
\begin{aligned}
& \gamma_{R}\left(P_{n} \square C_{3}\right)=\left\lfloor\frac{3 n}{2}\right\rfloor+1 \\
& \gamma_{R}\left(P_{n} \square C_{4}\right)= \begin{cases}3 ; & \text { if } n=1 \\
2 n ; & \text { otherwise }\end{cases} \\
& \gamma_{R}\left(P_{n} \square C_{5}\right)=2 n+2 \\
& \gamma_{R}\left(P_{n} \square C_{6}\right)=\left\lfloor\frac{8 n}{3}\right\rfloor+2 \\
& \gamma_{R}\left(P_{n} \square C_{7}\right)= \begin{cases}3 n+2 ; & \text { if } n \in\{1,2,4\} \\
3 n+3 ; & \text { otherwise }\end{cases} \\
& \gamma_{R}\left(P_{n} \square C_{8}\right)= \begin{cases}8 ; & \text { if } n=2 \\
\left\lfloor\frac{7 n}{2}\right\rfloor+2 ; & \text { if } n \in\{3,4,8\} \\
\left\lfloor\frac{7 n}{2}\right\rfloor+3 ; & \text { otherwise }\end{cases}
\end{aligned}
$$

\section{3 $\gamma_{R}\left(C_{n} \square P_{k}\right)$ for $k \in\{2,3,4,5,6\}$ and $n \geqslant 3$}

We implemented this case as a rotagraph. From (3) we know that the calculations for rotagraphs take much more time than the calculations for fasciagraphs. Therefore we covered only the cases for $k \in\{2,3,4,5,6\}$. As in former cases, formulas for the Roman domination number are presented below and constructions can be found in Figures 13 to 
18.

$$
\begin{aligned}
& \gamma_{R}\left(C_{n} \square P_{2}\right)= \begin{cases}n ; & \text { if } n \in\{4 k \mid k \in \mathbb{N}\} \\
n+1 ; & \text { otherwise }\end{cases} \\
& \gamma_{R}\left(C_{n} \square P_{3}\right)= \begin{cases}5 ; & n=3 \\
\left\lceil\frac{3 n}{2}\right\rceil ; & \text { if } n \in\{4 k, 4 k+1 \mid k \in \mathbb{N}\} \\
\left\lceil\frac{3 n}{2}\right\rceil+1 ; & \text { otherwise }\end{cases} \\
& \gamma_{R}\left(C_{n} \square P_{4}\right)= \begin{cases}7 ; & \text { if } n=3 \\
2 n ; & \text { otherwise }\end{cases} \\
& \gamma_{R}\left(C_{n} \square P_{5}\right)= \begin{cases}\left\lceil\frac{12 n}{5}\right\rceil+1 ; & \text { if } n \in\{5 k+2 \mid k \in \mathbb{N}\} \\
\left\lceil\frac{12 n}{5}\right\rceil ; & \text { otherwise }\end{cases} \\
& \gamma_{R}\left(C_{n} \square P_{6}\right)= \begin{cases}\left\lfloor\frac{14 n}{5}\right\rfloor ; & \text { if } n \in\{5 k \mid k \in \mathbb{N}\} \\
\left\lfloor\frac{14 n}{5}\right\rfloor+1 ; & \text { if } n \in\left\{5 k+4 \mid k \in \mathbb{N}_{0}\right\} \\
\left\lfloor\frac{14 n}{5}\right\rfloor+2 ; & \text { otherwise }\end{cases}
\end{aligned}
$$

\section{$5.4 \gamma_{R}\left(C_{n} \square C_{k}\right)$ for $k \in\{3,4,5,6\}$ and $n \geqslant 3$}

In [18], the authors showed that $\gamma_{R}\left(C_{5 n} \square C_{5 m}\right)=10 m n$, which is consistent with our calculations. We found no other formulas for the Roman domination numbers of graphs $C_{n} \square C_{k}$. Constructions for each case can be found in Figures 19 to 23.

$$
\begin{aligned}
& \gamma_{R}\left(C_{n} \square C_{3}\right)=\left\lceil\frac{3 n}{2}\right\rceil \\
& \gamma_{R}\left(C_{n} \square C_{4}\right)=2 n \\
& \gamma_{R}\left(C_{n} \square C_{5}\right)= \begin{cases}2 n ; & \text { if } n \in\{5 k \mid k \in \mathbb{N}\} \\
2 n+2 ; & \text { otherwise }\end{cases} \\
& \gamma_{R}\left(C_{n} \square C_{6}\right)= \begin{cases}\left\lfloor\frac{8 n}{3}\right\rfloor ; & \text { if } n \in\{6 k \mid k \in \mathbb{N}\} \\
\left\lfloor\frac{8 n}{3}\right\rfloor+1 ; & \text { if } n \in\left\{6 k+5,18 k+3,18 k+8,18 k+13 \mid k \in \mathbb{N}_{0}\right\} \\
\left\lfloor\frac{8 n}{3}\right\rfloor+2 ; & \text { otherwise }\end{cases}
\end{aligned}
$$

\section{Roman graphs}

Combining results obtained here and known results on the domination number of graphs $[1,8,19,35,42]$ we also looked for Roman graphs (graphs, satisfying $\gamma_{R}(G)=2 \gamma(G)$ ). In the cases where the domination numbers of graphs have not been calculated yet, we also 
refer to a simple observation: a graph $G$ cannot be a Roman graph if $\gamma_{R}(G)$ is an odd number. It is also known that a graph is a Roman graph if and only if it has a $\gamma_{R}$-function $f=\left(V_{0}, V_{1}, V_{2}\right)$ with $V_{1}=\emptyset\left([9]\right.$, Proposition 16). Except in cases $P_{n} \square C_{5}, P_{n} \square C_{8}, C_{n} \square P_{5}$, $C_{n} \square C_{5}$ and $C_{n} \square C_{6}$, the following is a complete listing of all Roman graphs in the classes of graphs that we have studied.

1. Roman graphs among $P_{n} \square P_{k}$ :

$$
\begin{array}{ll}
k=1: & n \in\left\{3 l+2,3 l+3 \mid l \in \mathbb{N}_{0}\right\} \\
k=2: & n \text { odd } \\
k=3: & n \in\{4 l+1,4 l+2,4 l+3 \mid l \in \mathbb{N}\} \\
k=4: & n \in \mathbb{N} \backslash\{1,2,3,5,6,9\} \\
k=5: & n \in\{1,2,3,7,5 l, 5 l+1 \mid l \in \mathbb{N}\} \\
k=6: & n \in\{1,3,5,7,8,12,15,22\} \\
k=7: & n \in\{2,3,4,5,6,7,8,10,11,13,16\} \\
k=8: & n \in\{1,4,6,7,8\}
\end{array}
$$

2. Roman graphs among $P_{n} \square C_{k}$ :

$k=3: \quad n \in\left\{4 l+1,4 l+2 \mid l \in \mathbb{N}_{0}\right\}$

$k=4: \quad n \geqslant 2$

$k=5: \quad n \in\{1,2,3\}$

$k=6: \quad n \in\{1,3,4,6,6 l+1,6 l+3,6 l+4,6 l+6 \mid l \in \mathbb{N}\}$

$k=7: \quad n \in\left\{2,4,2 l+1 \mid l \in \mathbb{N}_{0}\right\}$

$k=8: \quad n \in\{1,2,3,4,5,6\}$

3. Roman graphs among $C_{n} \square P_{k}$ :

$k=2: \quad n \in\{4 l, 4 l+1,4 l+3 \mid l \in \mathbb{N}\}$

$k=3: \quad n \geqslant 4$

$k=4: \quad n \in \mathbb{N} \backslash\{3,5,9\}$

$k=5: \quad n \in\{3,4,7,8,10 l, 10 l+4,10 l+7,10 l+8 \mid l \in \mathbb{N}\}$

$k=6: \quad n \in\{3,4,6,8,11,14,18,28\}$

4. Roman graphs among $C_{n} \square C_{k}$ :

$$
\begin{array}{ll}
k=3: & n \in\{4 l, 4 l+1 \mid l \in \mathbb{N}\} \\
k=4: & n \in \mathbb{N} \\
k=5: & n \in\{3,4,5 l, 5 l+1,5 l+2,5 l+4 \mid l \in \mathbb{N}\}
\end{array}
$$




$$
k=6: \quad n \in\{6 l, 6 l+4,18 l+1,18 l+5,18 l+7, \mid l \in \mathbb{N}\}
$$

Remark 6.1 It was proven in [42] that $n+\left\lceil\frac{n}{5}\right\rceil \leqslant \gamma\left(C_{n} \square P_{5}\right) \leqslant n+\left\lceil\frac{n}{4}\right\rceil$. In fact, we show here that since $C_{10 i} \square P_{5}, C_{10 i+4} \square P_{5}, C_{10 i+7} \square P_{5}$ and $C_{10 i+8} \square P_{5}$ are Roman graphs (see Figure 17), their domination number equals $\frac{\gamma_{R}}{2}$. More precisely,

- $\gamma_{R}\left(C_{10 i} \square P_{5}\right)=\left\lceil\frac{12(10 i)}{5}\right\rceil=24 i$ hence $\gamma\left(C_{10 i} \square P_{5}\right)=12 i=10 i+2 i=n+\frac{n}{5}$;

- $\gamma_{R}\left(C_{10 i+4} \square P_{5}\right)=\left\lceil\frac{12(10 i+4)}{5}\right\rceil=24 i+10$ hence $\gamma\left(C_{10 i+4} \square P_{5}\right)=12 i+5=(10 i+4)+$ $(2 i+1)=n+\left\lceil\frac{n}{5}\right\rceil$;

- $\gamma_{R}\left(C_{10 i+7} \square P_{5}\right)=\left\lceil\frac{12(10 i+7)}{5}\right\rceil=24 i+17+1=24 i+18$ hence $\gamma\left(C_{10 i+7} \square P_{5}\right)=$ $12 i+9=(10 i+7)+(2 i+2)=n+\left\lceil\frac{n}{5}\right\rceil ;$

- $\gamma_{R}\left(C_{10 i+8} \square P_{5}\right)=\left\lceil\frac{12(10 i+8)}{5}\right\rceil=24 i+20$ hence $\gamma\left(C_{10 i+8} \square P_{5}\right)=12 i+10=(10 i+$ $8)+(2 i+2)=n+\left\lceil\frac{n}{5}\right\rceil$.

\section{Summary and open problems}

Let us conclude with some remarks and suggestions for future work on this topic.

1. All the implementations of the algorithm presented here were performed on a personal computer or on a small computer cluster. Because of the time and space complexity limitations we could therefore not calculate the Roman domination numbers of the Cartesian products of paths and cycles for larger monographs although theoretically we can get the exact formula for $\gamma_{R}\left(P_{n} \square P_{k}\right)$ for any value of $k$. It would be interesting to find another way to determine $\gamma_{R}\left(P_{n} \square P_{k}\right)$ for any $k, n \in \mathbb{N}$. Recall that Chang's conjecture about the domination number of grid graphs was open for quite a long time before Gonçalves, Pinlou, Rao and Thomassé [19] proved it. Considering the fact that the nice expression $\gamma\left(P_{n} \square P_{k}\right)=\left\lfloor\frac{(k+2)(n+2)}{5}\right\rfloor-4$ holds for quite "large" grids, i.e. for $P_{n} \square P_{k}$ for any $16 \leqslant k \leqslant n$ we do not think we have enough information to make analogous conjecture for Roman domination on grid graphs. From the results that have been established, it seems that the Roman domination number of $P_{n} \square P_{k}$ could be of the form $\left\lfloor\frac{2(k+1) n}{5}\right\rfloor+C(k)$, where $C(k)$ is some constant, dependent of $k$.

2. As can be seen from $\gamma_{R}$-functions in Figures 3 to 23 , our graphs (except $C_{n} \square C_{6}$ for some $n$ ) have property that $V_{1} \cup V_{2}$ is an independent set. Note that in some cases two vertices of $V_{2}$ are adjacent in the figure because we wanted to show that they are Roman graphs. But in each case, one of the two adjacent vertices, which are assigned the value 2, can be replaced with two vertices of $V_{1}$ in a way that $V_{1} \cup V_{2}$ is an independent set. Such graphs were called Roman domination perfect graphs 
in [30] where it was shown that if a graph does not contain one of the forbidden induced subgraphs from a list, then it is Roman domination perfect graph. Note that all our graphs contain one of the forbidden induced subgraphs of that list, but are still Roman domination perfect graphs.

3. The same approach as used in this paper can provide algorithms for computing the Roman domination numbers of the other known graph products [22] of paths and cycles. Only bounds are known for the Roman domination number of the strong product of arbitrary graphs [51] and also $\gamma_{R}\left(P_{n} \otimes P_{k}\right)$ is not determined yet. To the best of our knowledge, no one has even tried to work on the Roman domination number of the direct product of graphs. The approach used here would make it possible to determine $\gamma_{R}\left(P_{n} \times P_{k}\right)$ for some fixed values of $k$, and more general results would be welcome. Roman domination number of the fourth well known graph product, the lexicographic product, was established in [37] using a new concept of the so-called dominating couples.

4. Several new graph invariants based on the Roman domination have been studied, for instance weak Roman domination [10, 27, 45], $k$-Roman domination [26], Roman $k$-domination [34] and others [3, 5, 13, 28]. We believe that a similar approach could yield constant time algorithms for most or even all of these graph invariants on fasciagraphs and rotagraphs.

\section{Acknowledgments}

The authors would like to thank anonymous reviewers for careful reading of the manuscript and for many suggestions that helped us improve the presentation considerably.

\section{References}

[1] S. Alanko, S. Crevals, A. Isopoussu, P. Östergård and V. Pettersson, Computing the domination number of grid graphs, The Electronic Journal of Combinatorics, 18 (2011).

[2] J. Arquilla and H. Fredricksen, "Graphing" an optimal grand strategy, Military Operations Research, Fall 1995.

[3] M. Atapour, S. M. Sheikholeslami and A. Khodkar, Roman domination subdivision number of graphs, Aequationes Mathematicae, 78 (2009), 237-245.

[4] M. Bouznif, J. Moncel and M. Preissmann, Generic algorithms for some decision problems on fasciagraphs and rotagraphs, Discrete Mathematics, 312 (2012), 27072719.

[5] A. P. Burger. E. J. Cockayne, W. R. Gründlingh, C. M. Mynhardt, J. H. van Vuuren and W. Winterbach, Finite order domination in graphs, Journal of Combinatorial Mathematics and Combinatorial Computing, 49 (2004), 159-175. 
[6] B. Carre, Graphs and Networks, Clarendon Press, Oxford, 1979.

[7] E. W. Chambers, B. Kinnersley, N. Price and D. B. West, Extremal problems for Roman domination, SIAM Journal on Discrete Mathematics, 23(3) (2009), 15751586.

[8] T. Y. Chang, W. E. Clark and E. O. Hare, Domination numbers of complete grid graphs, I, Ars Combinatoria, 38 (1994), 97-11.

[9] E. J. Cockayne, P. A. Dreyer, S. M. Hedetniemi and S. T. Hedetniemi, Roman domination in graphs, Discrete Mathematics, 278 (2004), 11-22.

[10] E. J. Cockayne, P. J. P. Grobler, W. R. Gründlingh, J. Munganga and J. H. van Vuuren, Protection of a graph, Utilitas Mathematica, 67 (2005), 19-32.

[11] E. J. Cockayne, E. O. Hare, S. T. Hedetniemi and T. Wymer, Bounds for the domination number of grid graphs, Congressus Numerantium, 47 (1985), 217-228.

[12] P. A. Dreyer, Applications and Variations of Domination in Graphs, PhD thesis, Rutgers University, New Jersey, 2000.

[13] E. Ebrahimi Targhi, N. Jafari Rad and L. Volkmann, Unique response Roman domination in graphs, Discrete Applied Mathematics, 159 (2011), 1110-1117.

[14] M. H. El-Zahar, S. M. Khamis and Kh. M. Nazzal, On the domination number of the Cartesian product of the cycle of length $n$ and any graph, Discrete Applied Mathematics, 155 (2007), 515-522.

[15] O. Favaron, H. Karami, R. Khoeilar and S.M. Sheikholeslami, Note on the Roman domination number of a graph, Discrete Mathematics 309 (2009), 3447-3451.

[16] H. Fernau, Roman domination: a parameterized perspective, International Journal of Computer Mathematics, 85 (1) (2008), 25-38.

[17] D. C. Fisher, The domination number of complete grid graphs, manuscript, 1993.

[18] X. Fu, Y. Yang and B. Jiang, Roman domination in regular graphs, Discrete Mathematics, 309 (2009), 1528-1537.

[19] D. Gonçalves, A. Pinlou, M. Rao and S. Thomassé, The domination number of grids, SIAM Journal on Discrete Mathematics, 25 (3) (2011), 1443-1453.

[20] S. Gravier and M. Mollard, Note on domination numbers of Cartesian product of paths, Discrete Applied Mathematics, 80 (1997), 247-250.

[21] W. Günther, The automorphism group of roto- and fasciagraphs, MATCH Communications in Mathematical and in Computer Chemistry, 14 (1983), 3-42.

[22] R. Hammack, W. Imrich and S. Klavžar, Handbook of Product Graphs, CRC Press, Boca Raton, London, New York, 2011.

[23] T. W. Haynes, S. T. Hedetniemi and P. J. Slater, Domination in Graphs: Advanced Topics, Marcel Dekker, New York, 1998.

[24] T. W. Haynes, S. T. Hedetniemi and P. J. Slater, Fundamentals of Domination in Graphs, Marcel Dekker, New York, 1998. 
[25] M. A. Henning, A characterization of Roman trees, Discussiones Mathematicae Graph Theory, 22 (2002), 225-234.

[26] M. A. Hening, Defending the Roman empire from multiple attacks, Discrete Mathematics, 271 (2003), 101-115.

[27] M. A. Hening and S. T. Hedetniemi, Defending the Roman empire - A new strategy, Discrete Mathematics, 266 (2003), 239-251.

[28] N. Jafari Rad and L. Volkmann, On the Roman bondage number of planar graphs, Graphs and Combinatorics, 27 (2011), 531-538.

[29] N. Jafari Rad and L. Volkmann, Roman domination dot-critical graphs, Graphs and Combinatorics, DOI 10.1007/s00373-012-1139-1.

[30] N. Jafari Rad and L. Volkmann, Roman domination perfect graphs, Analele Stiintifice ale Universitatii Ovidius Constanta, 19 (3) (2011), 167-174.

[31] M. Juvan, B. Mohar, A. Graovac, S. Klavžar and J. Žerovnik, Fast computation of the Wiener index of fasciagraphs and rotagraphs, Journal of Chemical Information and Computer Sciences, 35 (5) (1995), 834-840.

[32] M. Juvan, B. Mohar and J. Žerovnik, Distance-related invariants on polygraphs, Discrete Applied Mathematics, 80 (1997), 57-71.

[33] M. Juvan, M. Petkovšek, A. Graovac, A. Vesel and J. Žerovnik, The Szeged index of fasciagraphs, MATCH Communication in Mathematical and in Computer Chemistry, 49 (2003), 47-66.

[34] K. Kämmerling and L. Volkmann, Roman k-domination in graphs, Journal of the Korean Mathematical Society, 46 (6) (2009), 1309-1318.

[35] S. Klavžar and N. Seifter, Dominating Cartesian products of cycles, Discrete Applied Mathematics, 59 (1995), 129-136.

[36] S. Klavžar and J. Žerovnik, Algebraic approach to fasciagraphs and rotagraphs, Discrete Applied Mathematics, 68 (1996), 93-100.

[37] T. Kraner Šumenjak, P. Pavlič and A. Tepeh, On the Roman domination in the lexicographic product of graphs, Discrete Applied Mathematics, 160 (13-14) (2012), 2030-2036.

[38] M. Liedloff, T. Kloks, J. Liu and S.-L. Peng, Efficient algorithms on Roman domination on some classes of graphs, Discrete Applied Mathematics, 156 (2008), 3400-3415.

[39] M. Liedloff, T. Kloks, J. Liu and S.-L. Peng, Roman domination over some graph classes, Lecture Notes in Computer Science, 3787 (2005), 103-114.

[40] M. Livingston and Q. Stout, Constant time computation of minimum dominating sets, Congressus Numerantium, 105 (1994), 116-128.

[41] B.P. Mobaraky and S.M. Sheikholeslami, Bounds on Roman domination numbers of graphs, Matematiki vesnik, 60 (2008), 247-253.

[42] M. Nandi, S. Parui and A. Adhikari, The domination numbers of cylindrical grid graphs, Applied Mathematics and Computation, 217 (2011), 4879-4889. 
[43] C. S. ReVelle and K.E. Rosing, Defendens Imperium Romanum: a classical problem in military strategy, The American Mathematical Monthly, 107 (7) (2000), 585-594.

[44] F. Romani, Shortest-path problem is not harder than matrix multiplication, Information Processing Letters, 11 (3) (1980), 134-136.

[45] P. R. L. Pushpam and T. N. M. Malini Mai, Weak Roman domination in graphs, Discussiones Mathematicae Graph Theory, 31 (2011), 115-128.

[46] W. Shang and X. Hu, The Roman domination problem in unit disk graphs, Lecture Notes in Computer Science, 4489 (2007), 305-312.

[47] X. Song and W. Shang, Roman domination in a tree, Ars Combinatoria, 98 (2011), $73-82$.

[48] I. Stewart, Defend the Roman Empire!, Scientific American, 281 (6) (1999), 136-138.

[49] Y. Wu, An Improvement on Vizing's conjecture, manuscript, 2009.

[50] H.-M. Xing, X. Chen and X.-G. Chen, A note on Roman domination in graphs, Discrete Mathematics, 306 (2006), 3338-3340.

[51] I. G. Yero and J. A. Rodríguez-Velázquez, Roman domination in Cartesian product graphs and strong product graphs, manuscript, 2011.

[52] X. Zhang, J. Liu, X. Chen and J. Meng, Domination number of Cartesian products of directed cycles, Information Processing Letters, 111 (2010), 36-39.

[53] J. Žerovnik, Deriving formulas for domination numbers of fasciagraphs and rotagraphs, Lecture Notes in Computer Science, 1684 (1999), 559-568.

[54] J. Žerovnik, New formulas for the pentomino exclusion problem, Australasian Journal of Combinatorics, 36 (2006), 197-212. 

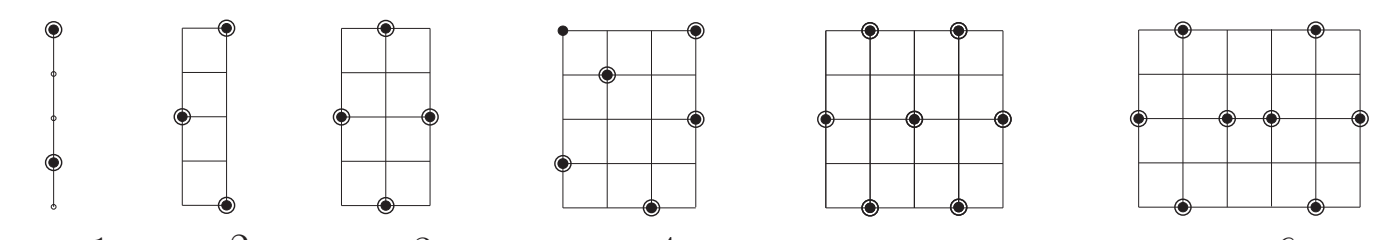

$n=1 \quad n=2 \quad n=3$

$n=4$

$n=5$

$n=6$

$n=5 k+2$

$\gamma_{R}=12 k+6$
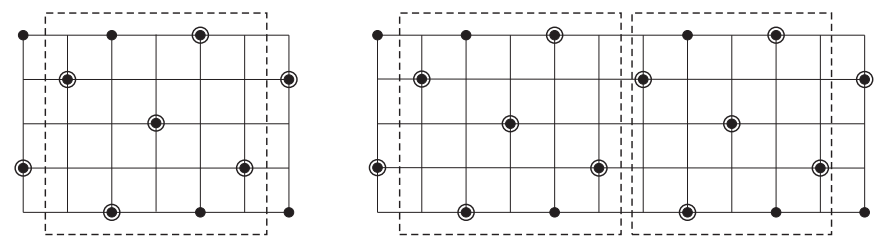

$n=5 k+3$

$\gamma_{R}=12 k+9$
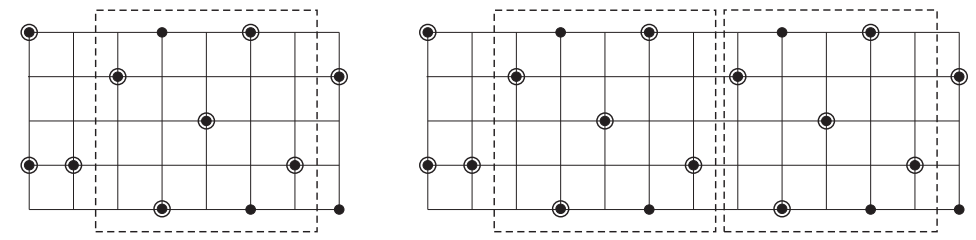

$n=5 k+4$

$\gamma_{R}=12 k+11$
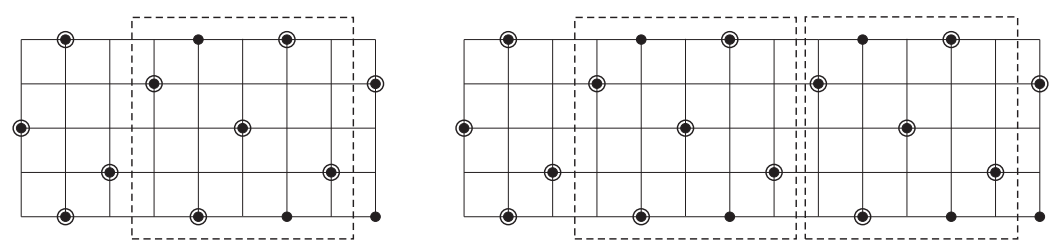

$n=5 k$

$\gamma_{R}=12 k+2$
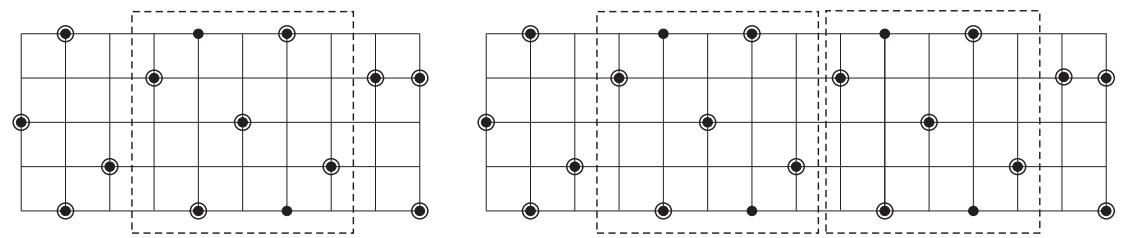

$n=5 k+1$

$\gamma_{R}=12 k+4$
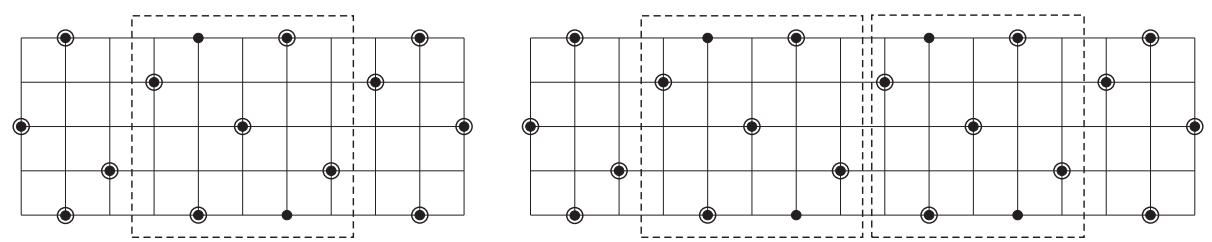

Figure 3: $P_{n} \square P_{5}$ 

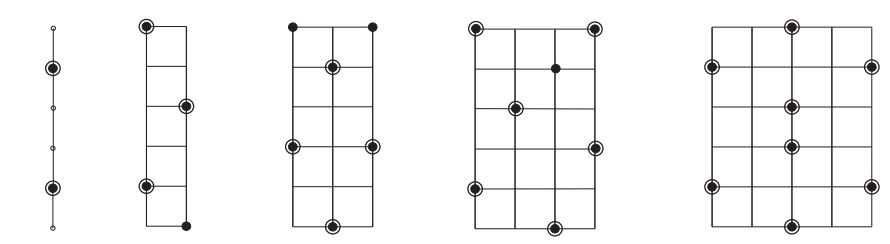

$n=1 n=2$

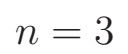

$n=4$

$n=5$

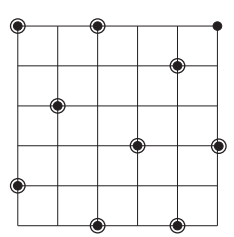

$n=6$

$n=5 k+2$

$\gamma_{R}=14 k+8$
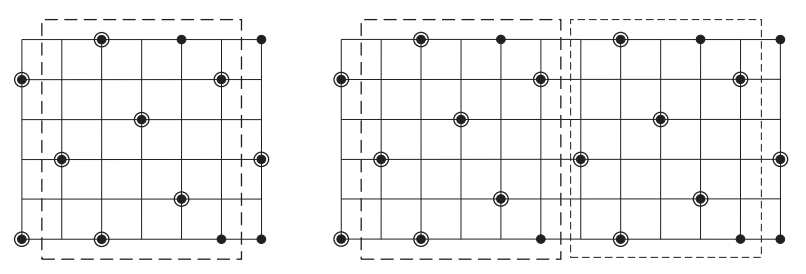

$n=5 k+3$

$\gamma_{R}=14 k+10$
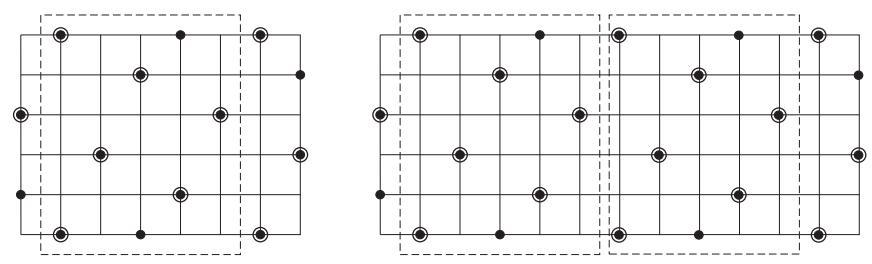

$n=5 k+4$

$\gamma_{R}=14 k+13$
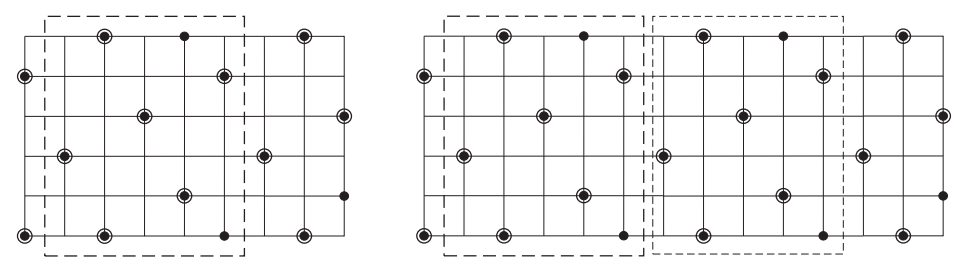

$n=5 k$

$\gamma_{R}=14 k+2$
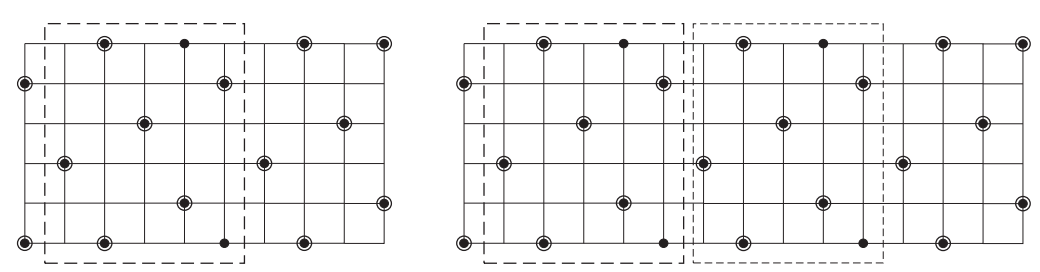

$n=5 k+1$

$\gamma_{R}=14 k+5$
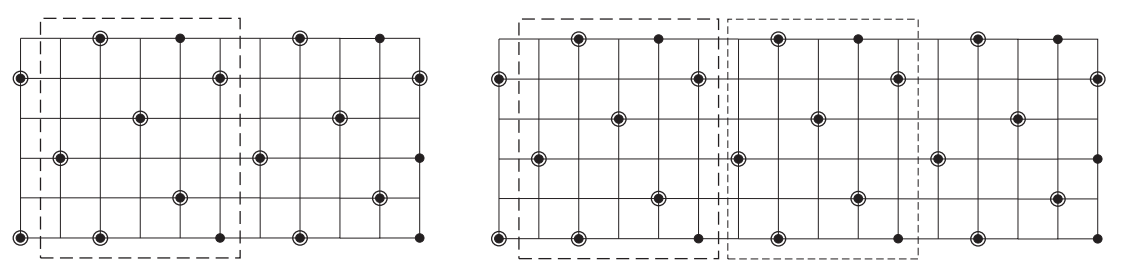

Figure 4: $P_{n} \square P_{6}$ 

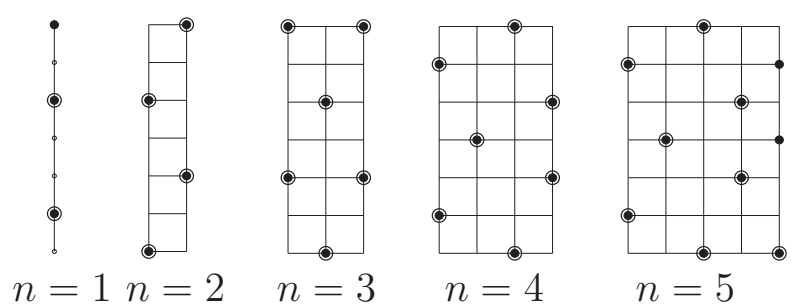

$n=5$

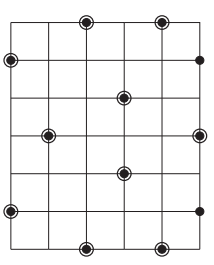

$n=6$

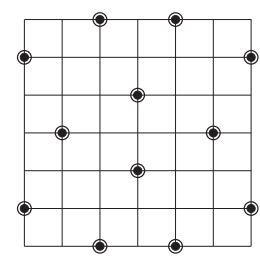

$n=7$

$n=5 k+3$

$\gamma_{R}=16 k+12$
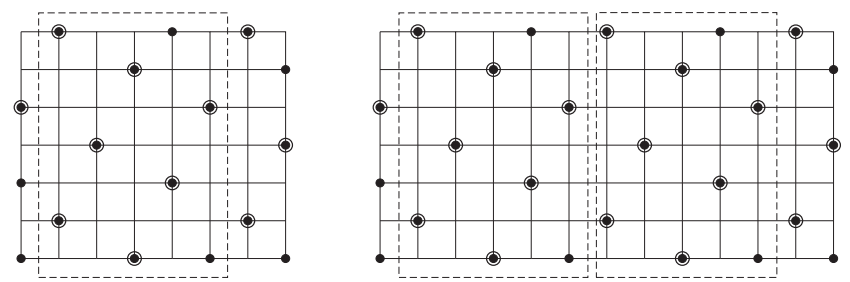

$n=5 k+4$

$\gamma_{R}=16 k+15$
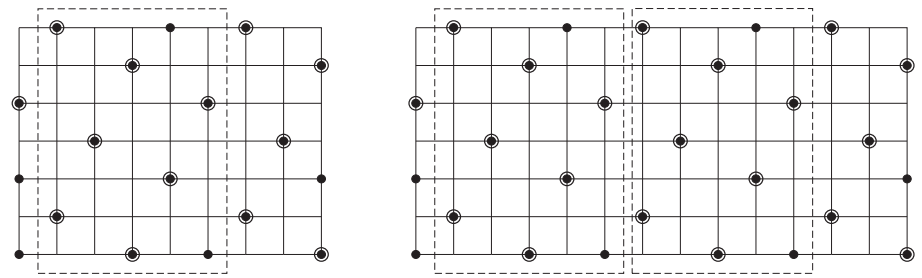

$n=5 k$

$\gamma_{R}=16 k+2$
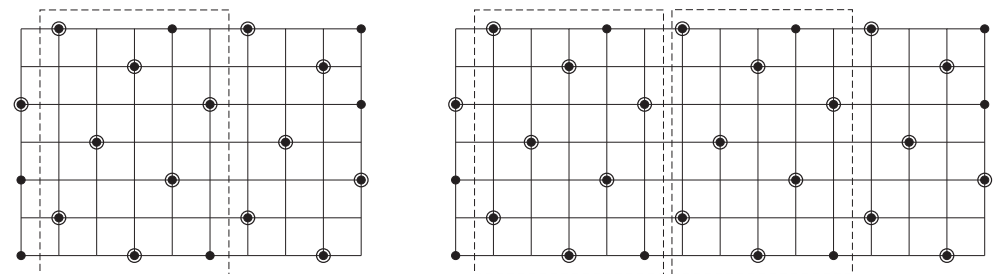

$n=5 k+1$

$\gamma_{R}=16 k+6$
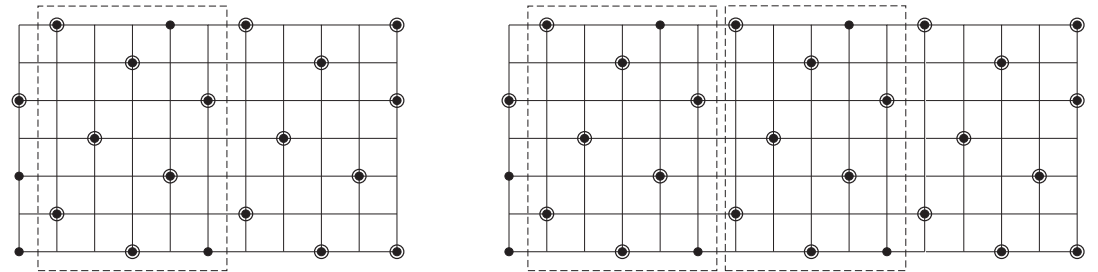

$n=5 k+2$

$\gamma_{R}=16 k+9$
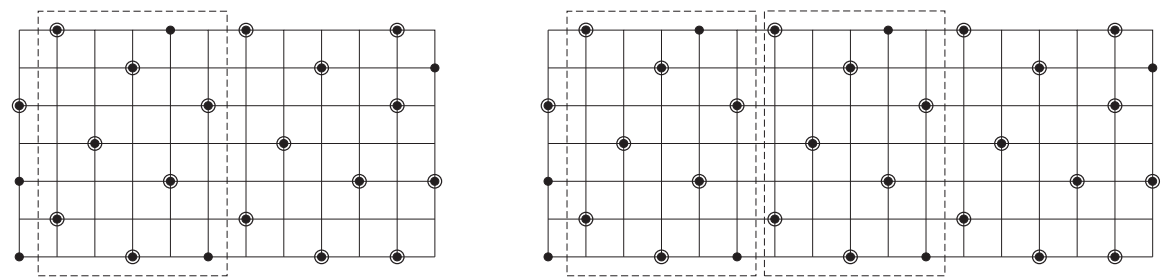

Figure 5: $P_{n} \square P_{7}$ 

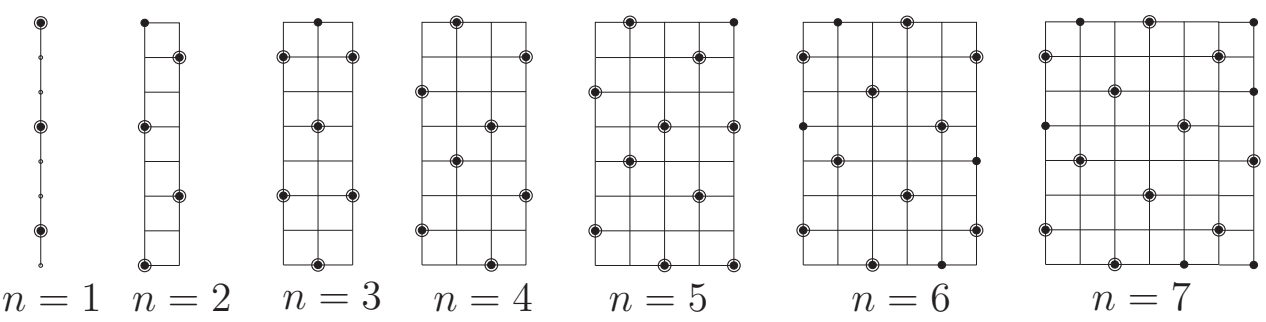

$$
n=5 \quad n=6 \quad n=7
$$

$$
\begin{aligned}
& n=5 k+3 \\
& \gamma_{R}=18 k+14
\end{aligned}
$$
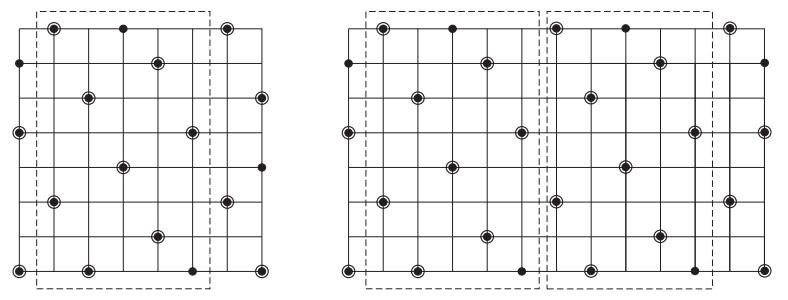

$$
\begin{aligned}
& n=5 k+4 \\
& \gamma_{R}=18 k+17
\end{aligned}
$$
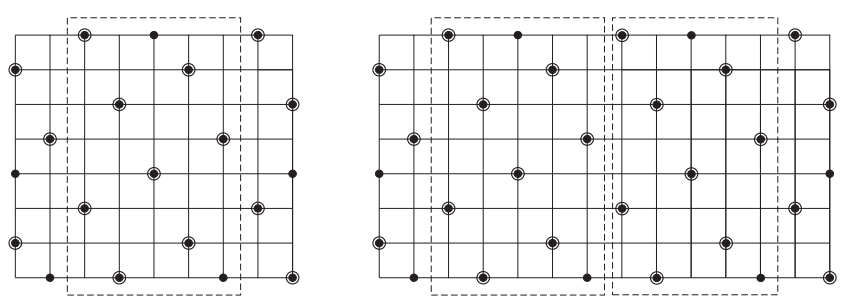

$$
\begin{aligned}
& n=5 k \\
& \gamma_{R}=18 k+3
\end{aligned}
$$
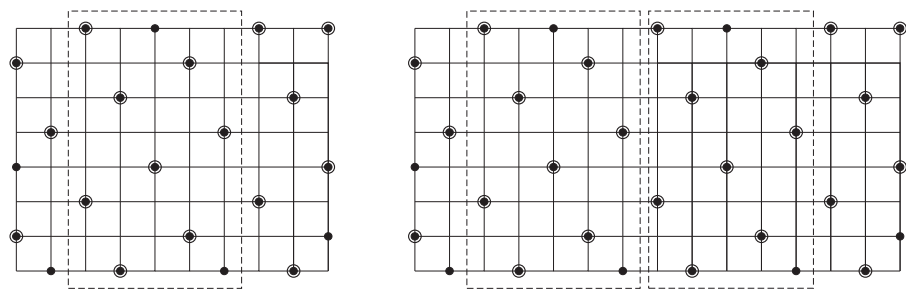

$n=5 k+1$

$\gamma_{R}=18 k+6$
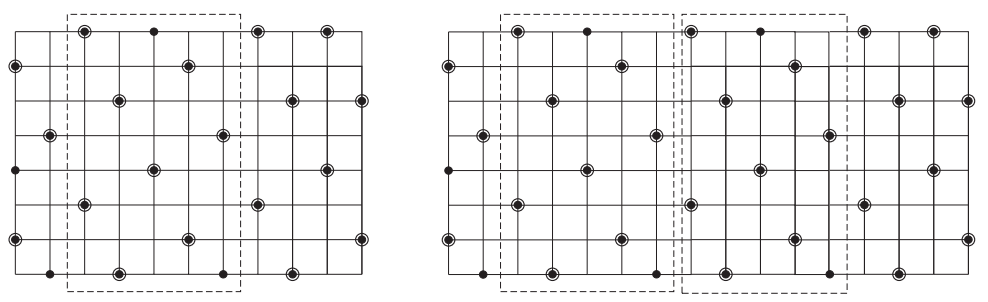

$$
\begin{aligned}
& n=5 k+2 \\
& \gamma_{R}=18 k+10
\end{aligned}
$$
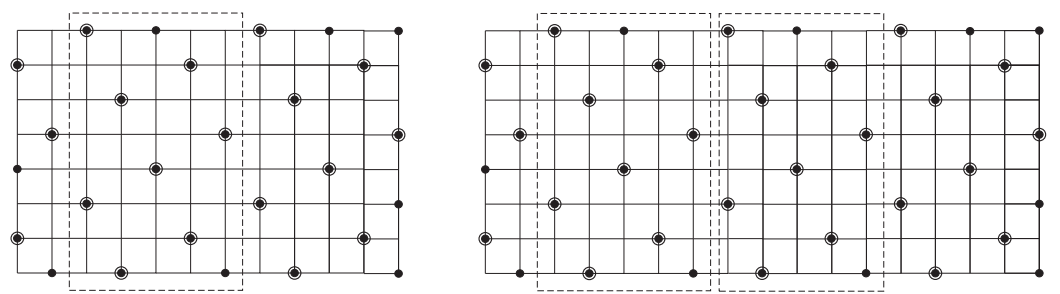

Figure 6: $P_{n} \square P_{8}$ 

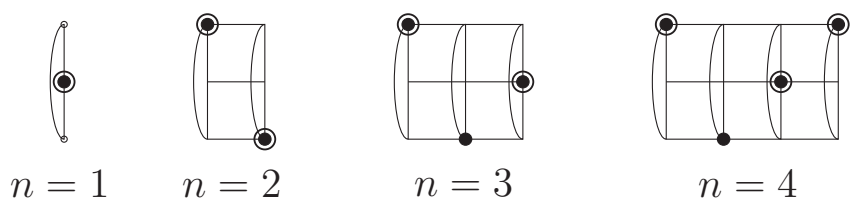

$n=4$

$n=4 k+1$
$\gamma_{R}=6 k+2$
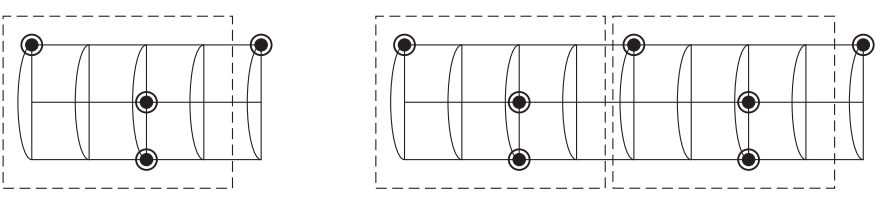

$$
\begin{gathered}
n=4 k+2 \\
\gamma_{R}=6 k+4
\end{gathered}
$$
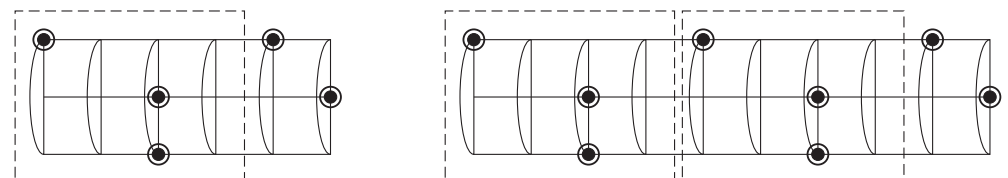

$$
\begin{gathered}
n=4 k+3 \\
\gamma_{R}=6 k+5
\end{gathered}
$$
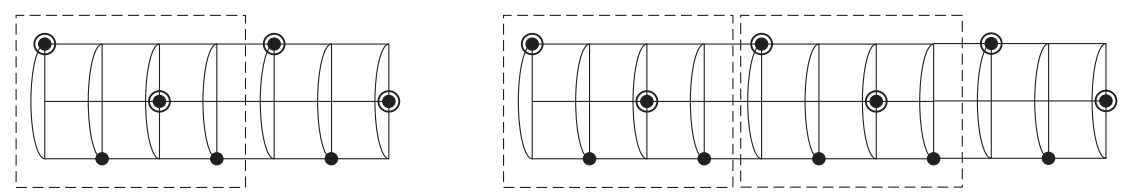

$$
\begin{aligned}
& n=4 k \\
& \gamma_{R}=6 k+1
\end{aligned}
$$
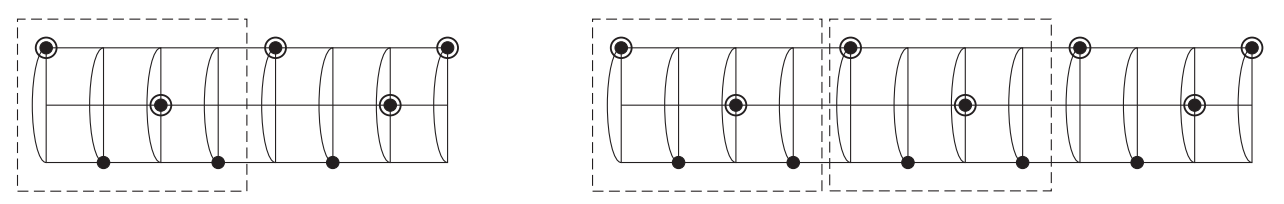

Figure 7: $P_{n} \square C_{3}$
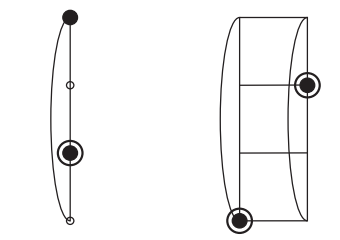

$n=1$

$n=2$

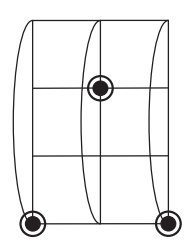

$n=3$

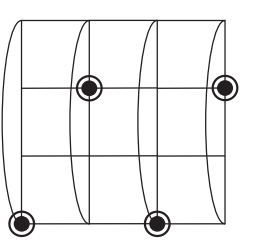

$n=4$

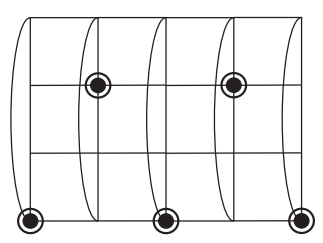

$n=5$

Figure 8: $P_{n} \square C_{4}$ 

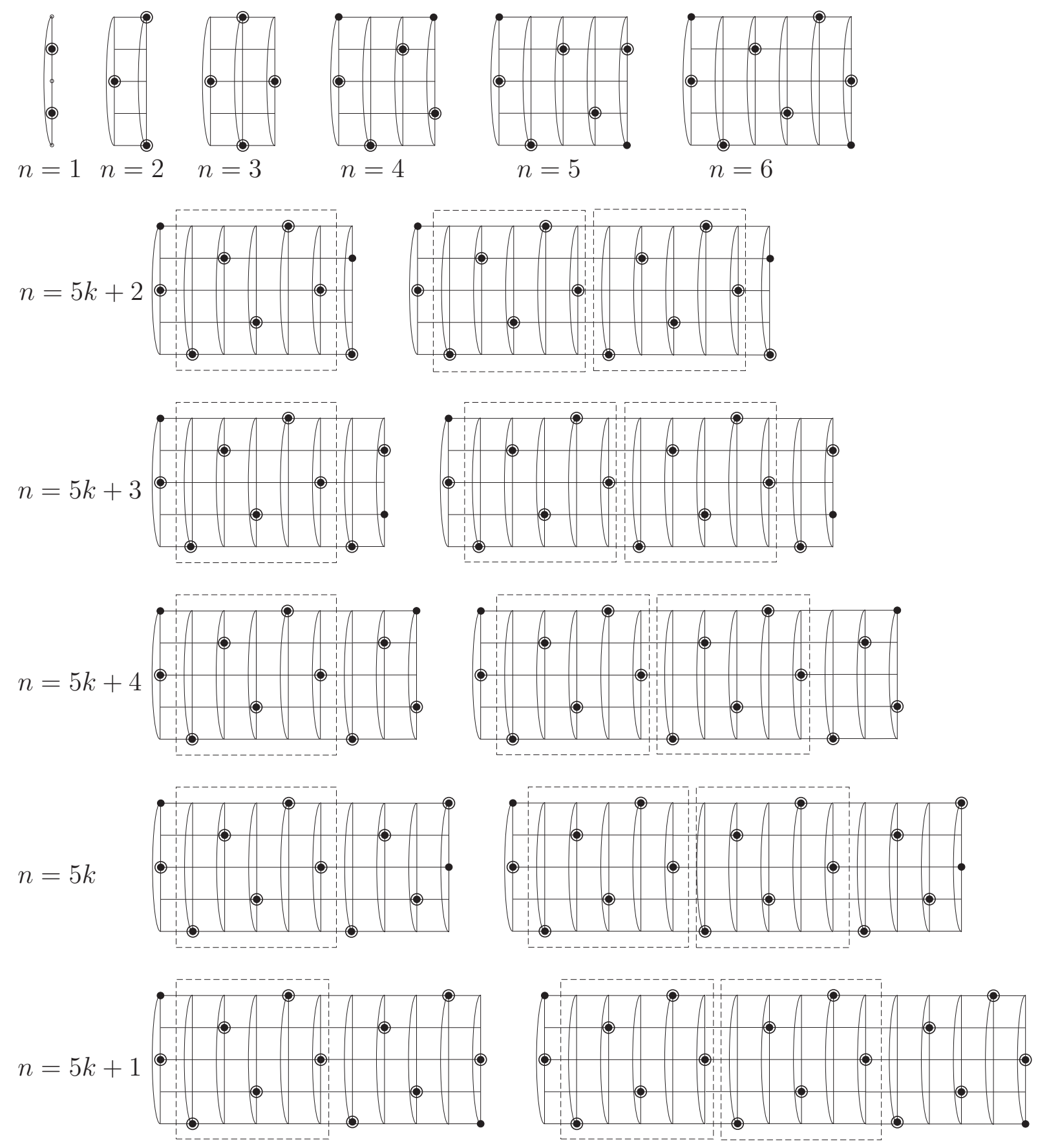

Figure 9: $P_{n} \square C_{5}$ 

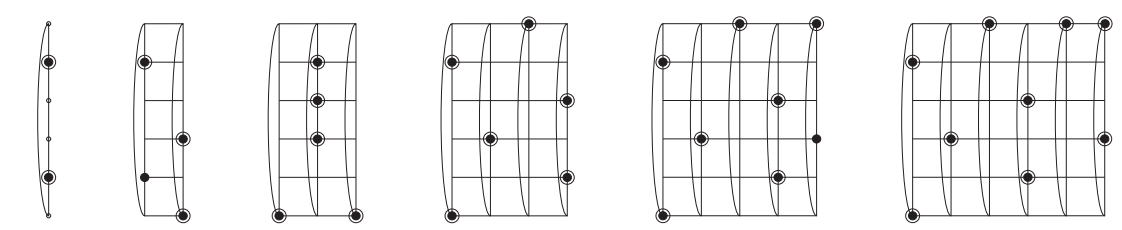

$n=1 n=2$

$n=3$

$n=4$

$n=5$

$n=6$

$n=6 k+1$

$\gamma_{R}=16 k+4$
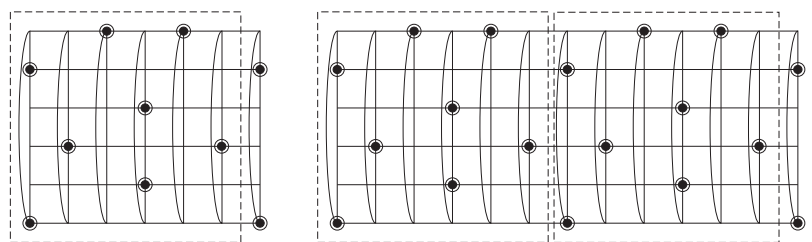

$n=6 k+2$

$\gamma_{R}=16 k+7$
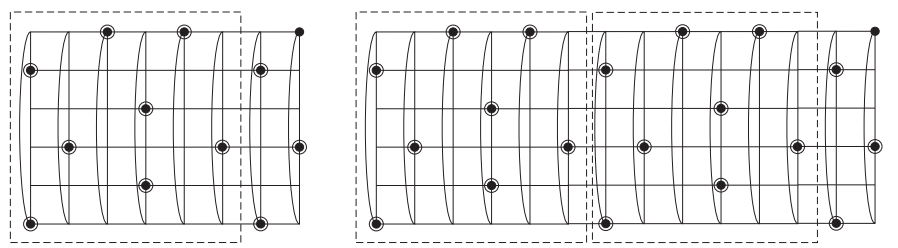

$n=6 k+3$

$\gamma_{R}=16 k+10$
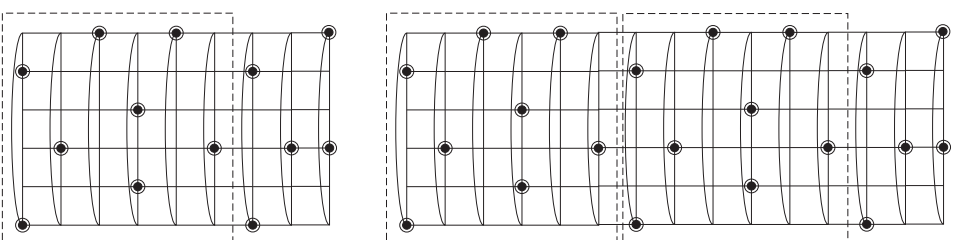

$n=6 k+4$

$\gamma_{R}=16 k+12$
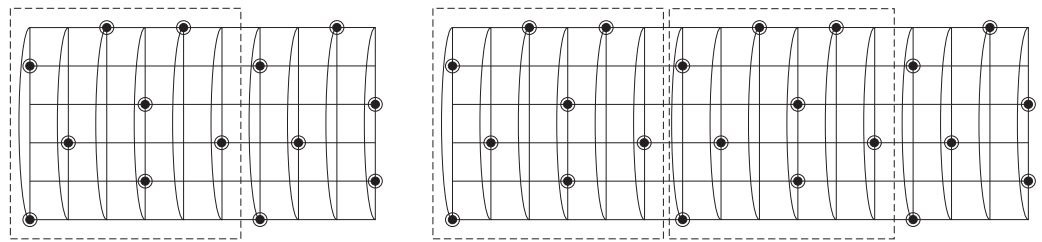

$n=6 k+5$

$\gamma_{R}=16 k+15$
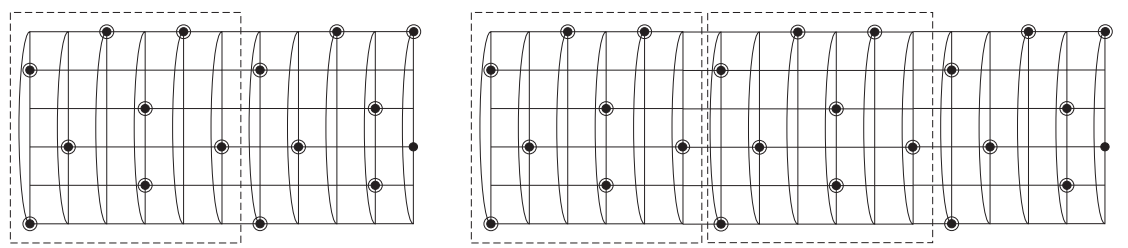

$n=6 k+6$

$\gamma_{R}=16 k+2$
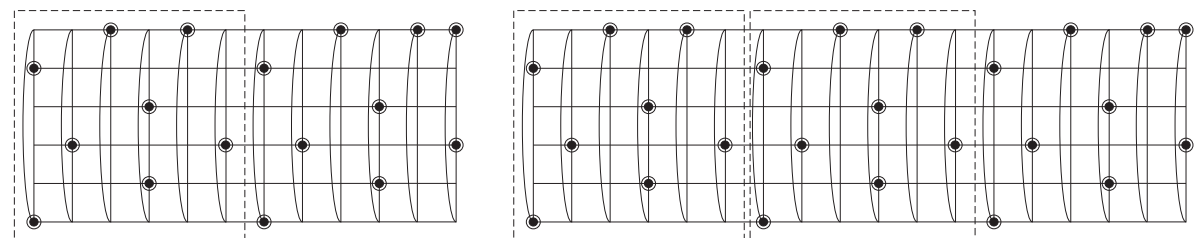

Figure 10: $P_{n} \square C_{6}$ 

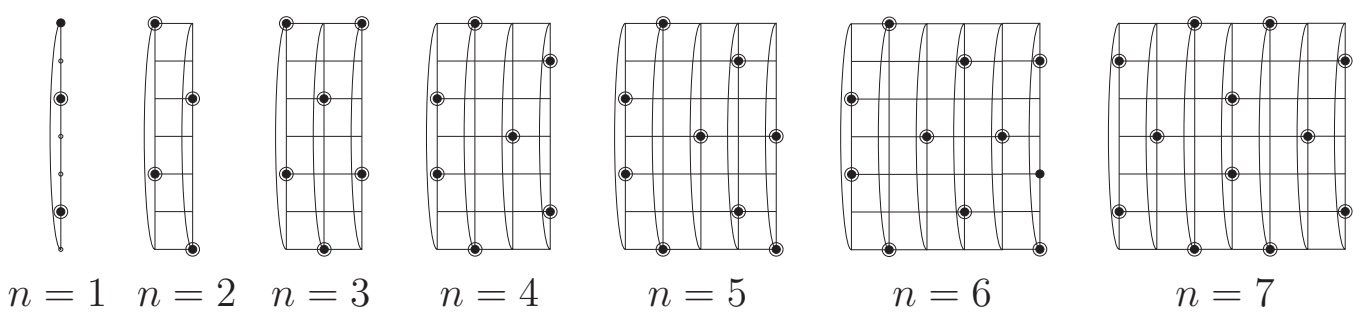

$$
n=6
$$

$$
n=7
$$

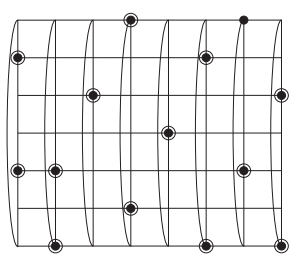

$n=8$

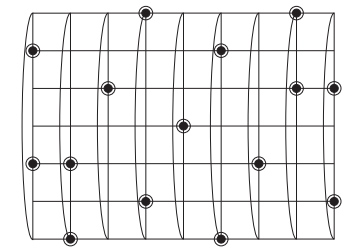

$n=9$

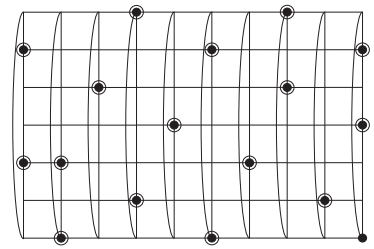

$n=10$

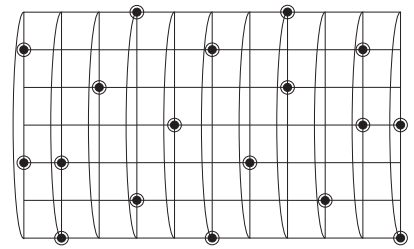

$n=11$

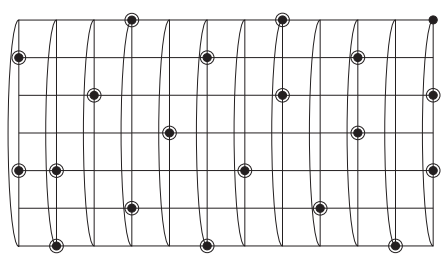

$n=12$

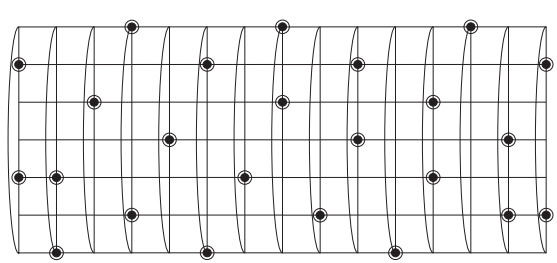

$n=15$

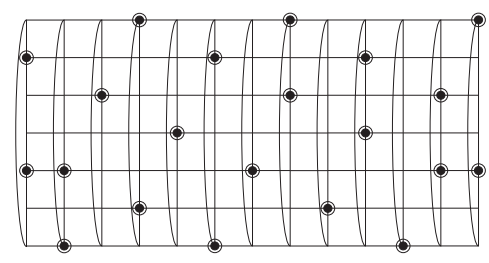

$n=13$

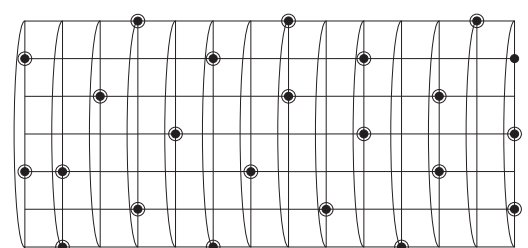

$n=14$

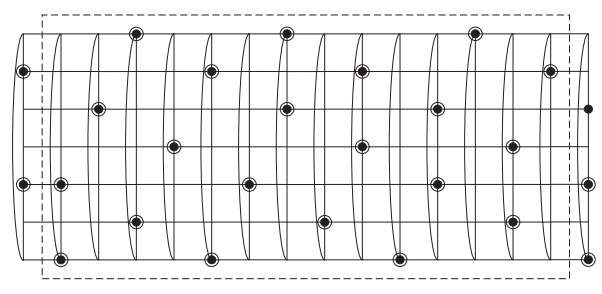

$$
n=14 k+2, \gamma_{R}=42 k+9
$$

Figure 11: $P_{n} \square C_{7}$ 

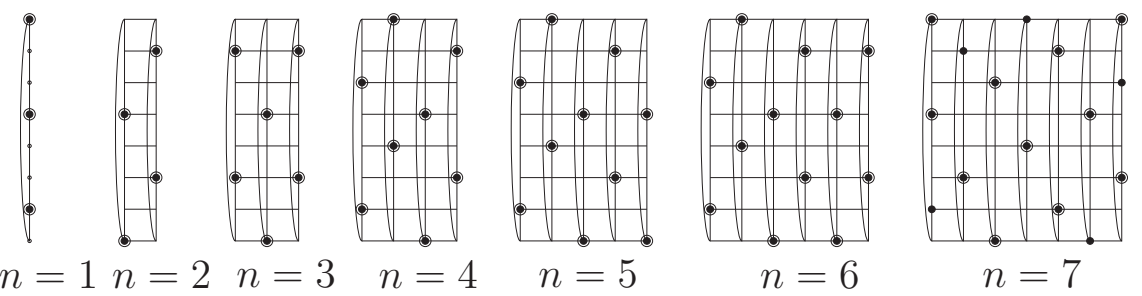

$n=6$

$n=7$

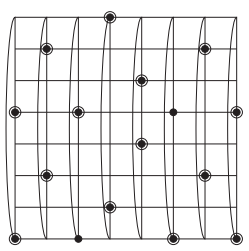

$n=8$

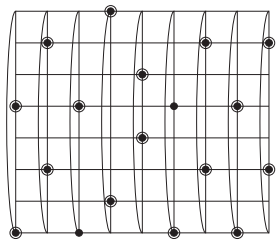

$n=9$

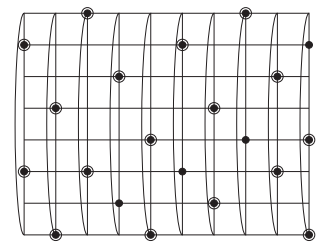

$n=10$

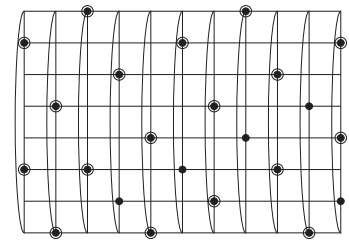

$n=11$

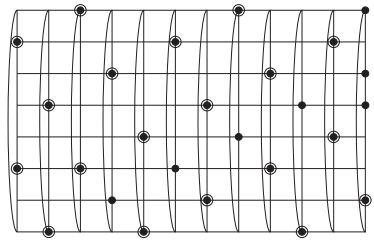

$n=12$

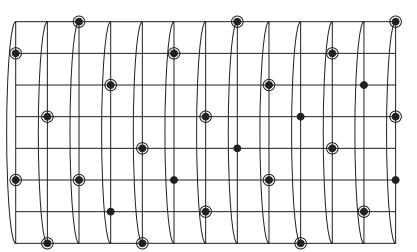

$n=13$

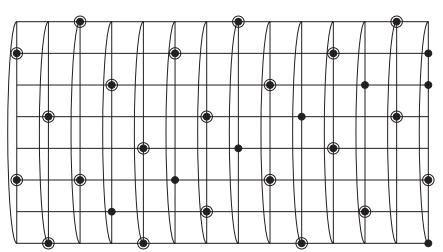

$n=14$

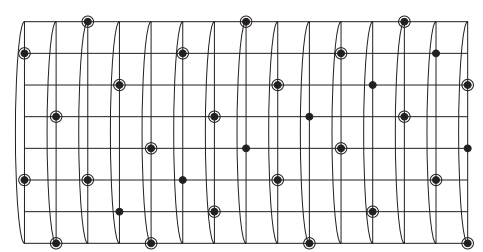

$n=15$

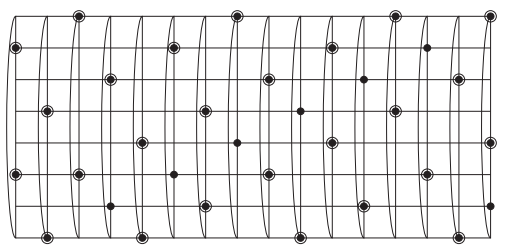

$n=16$

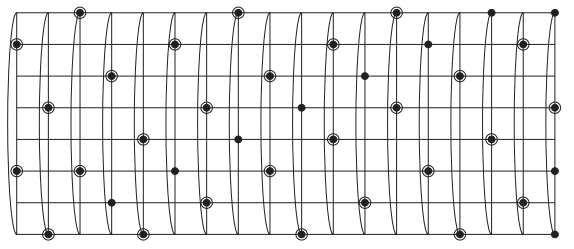

$n=18$

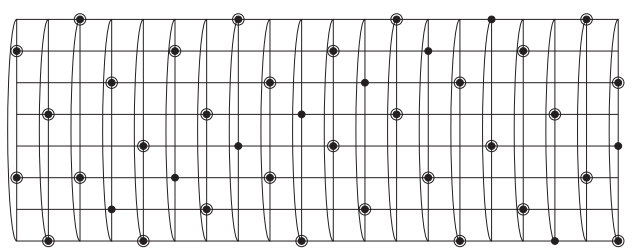

$n=20$

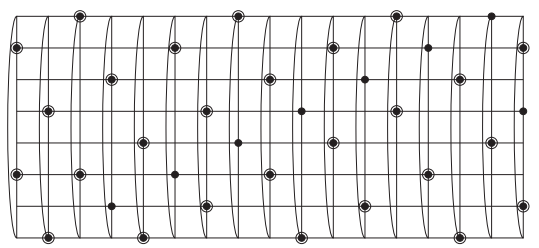

$n=17$

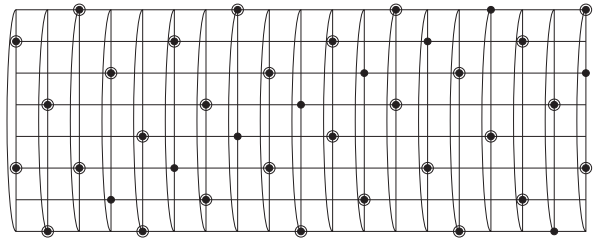

$n=19$

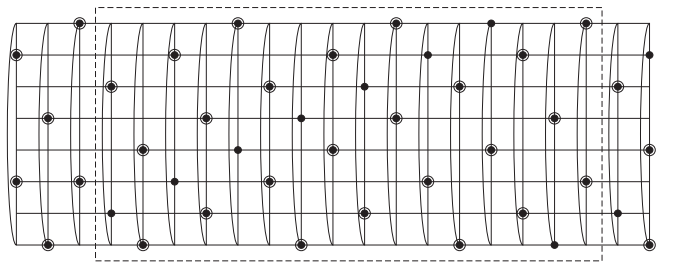

$n=16 k+5, \gamma_{R}=56 k+20$

Figure 12: $P_{n} \square C_{8}$ 


$$
\begin{gathered}
n=4 k \\
\gamma_{R}=4 k
\end{gathered}
$$
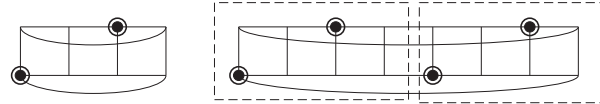

$$
\begin{aligned}
& n=4 k+1 \\
& \gamma_{R}=4 k+2
\end{aligned}
$$
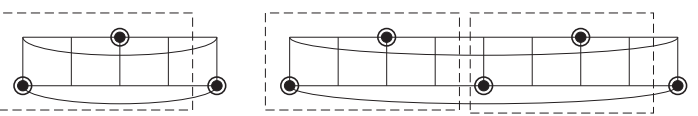

$n=4 k+2$
$\gamma_{R}=4 k+3$
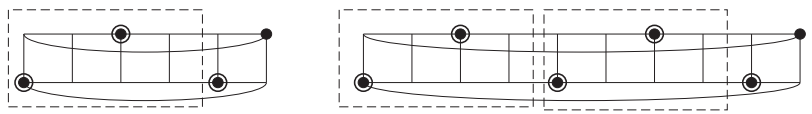

$$
\begin{gathered}
n=4 k+3 \\
\gamma_{R}=4 k+4
\end{gathered}
$$
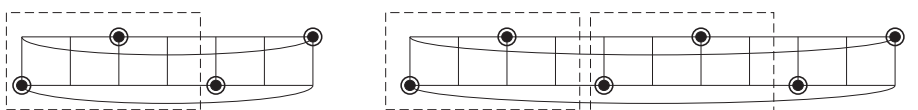

Figure 13: $C_{n} \square P_{2}$
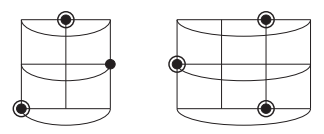

$n=3$

$n=4$

$n=4 k+1$

$\gamma_{R}=6 k+2$
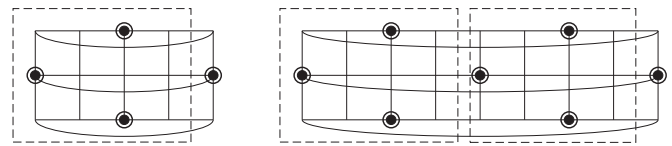

$n=4 k+2$
$\gamma_{R}=6 k+4$
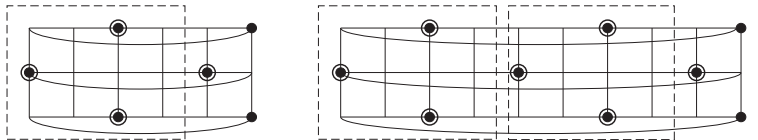

$n=4 k+3$

$\gamma_{R}=6 k+6$
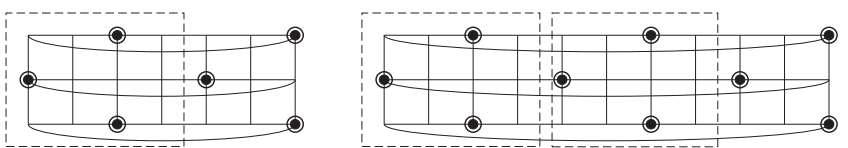

$$
\begin{aligned}
& n=4 k+4 \\
& \gamma_{R}=6 k+6
\end{aligned}
$$
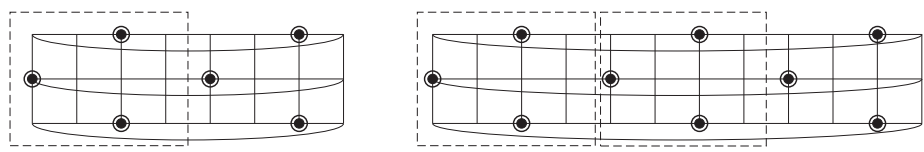

Figure 14: $C_{n} \square P_{3}$ 


$$
\begin{gathered}
n=4 k \\
\gamma_{R}=8 k
\end{gathered}
$$
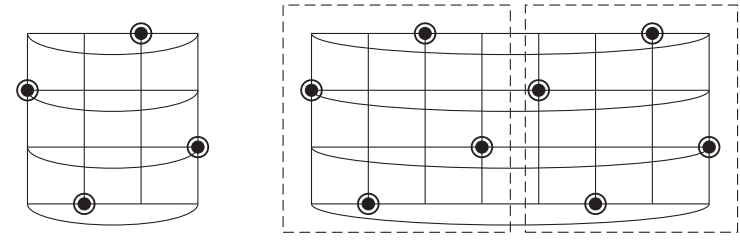

$n=4 k+1$

$\gamma_{R}=8 k+2$
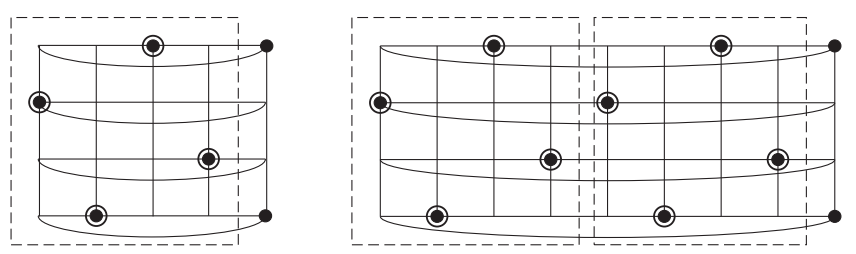

$n=4 k+2$

$\gamma_{R}=8 k+4$
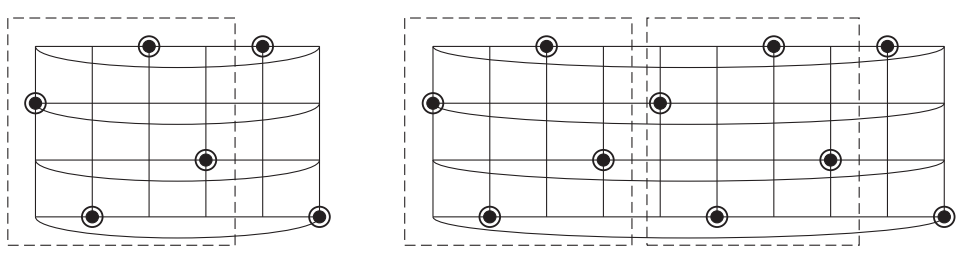

$n=4 k+3$

$\gamma_{R}=8 k+6$
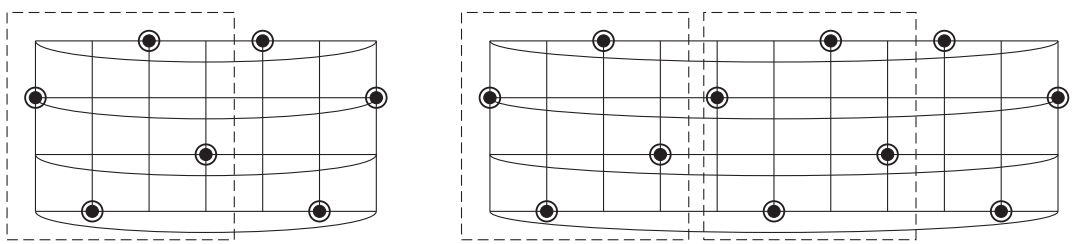

Figure 15: $C_{n} \square P_{4}$ 

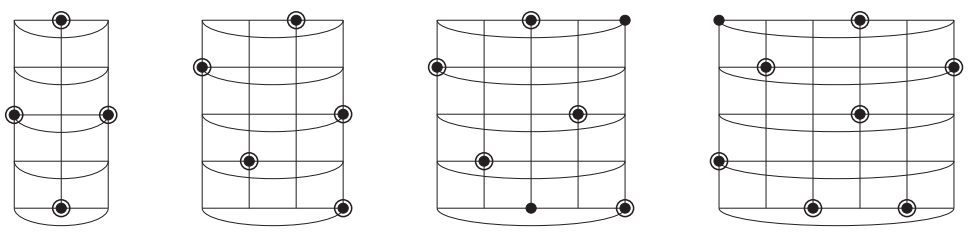

$n=3$

$n=4$

$n=5$

$n=6$

$n=5 k+2$

$\gamma_{R}=12 k+6$
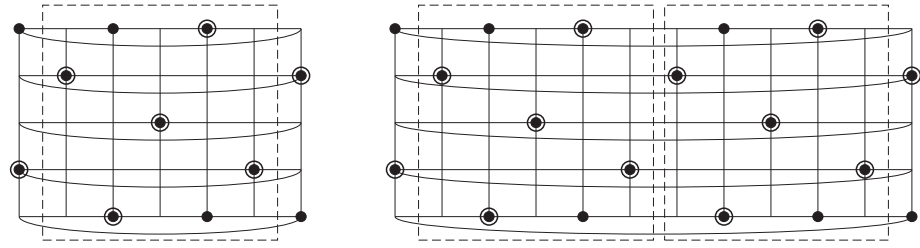

$n=5 k+3$

$\gamma_{R}=12 k+8$
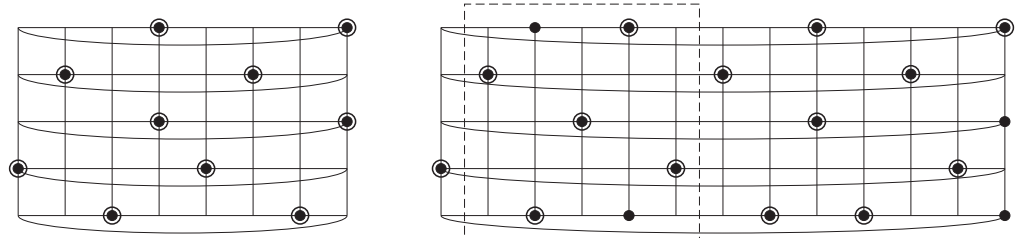

$n=5 k+4$

$\gamma_{R}=12 k+10$
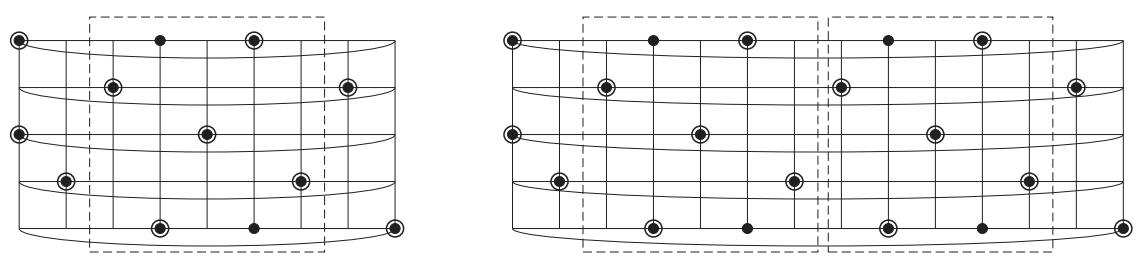

$n=5 k+5$

$\gamma_{R}=12 k+12$
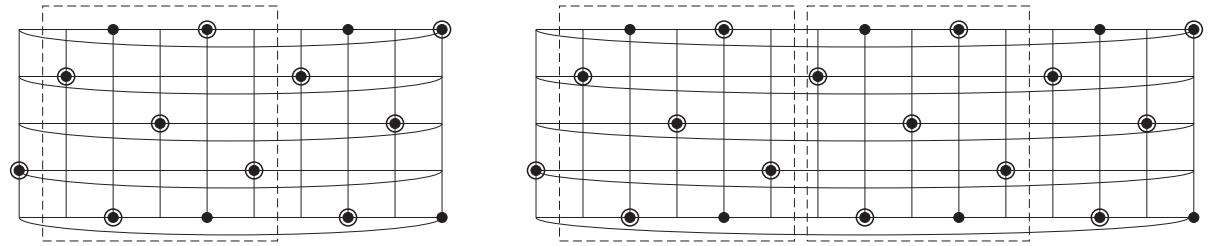

$n=5 k+1$

$\gamma_{R}=12 k+3$
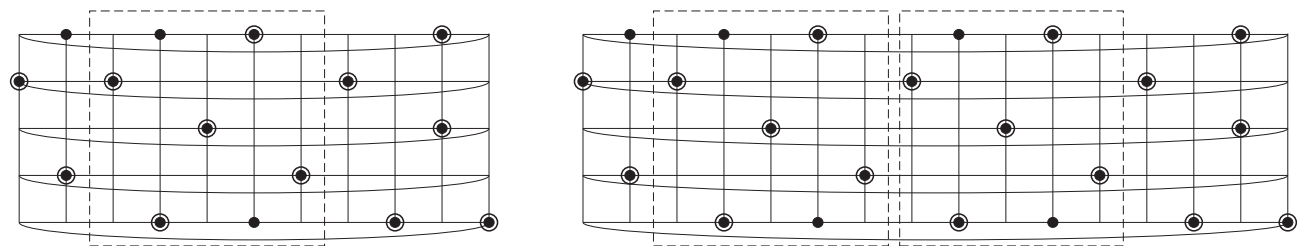

Figure 16: $C_{n} \square P_{5}$ 
$n=10 k$

$\gamma_{R}=24 k$
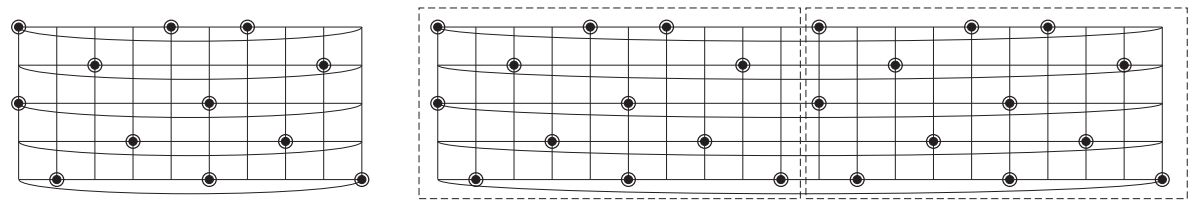

$n=10 k+4$

$\gamma_{R}=24 k+8$

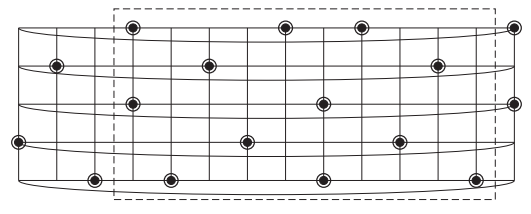

$n=10 k+7$

$\gamma_{R}=24 k+18$

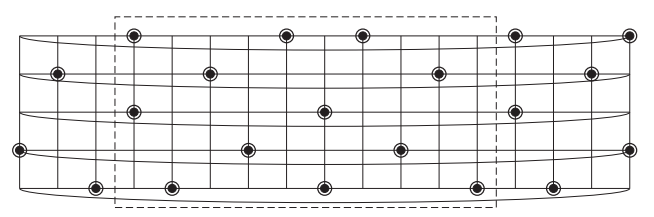

$n=10 k+8$

$\gamma_{R}=24 k+20$

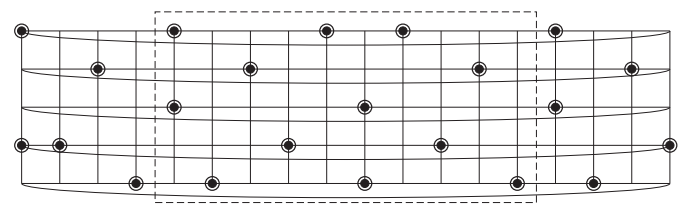

Figure 17: $C_{10 k} \square P_{5}, C_{10 k+4} \square P_{5}, C_{10 k+7} \square P_{5}$ and $C_{10 k+8} \square P_{5}$ are Roman graphs. 


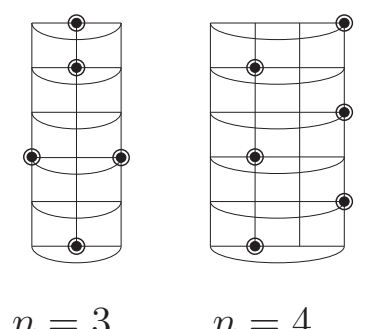

$n=3 \quad n=4$

$$
\begin{aligned}
& n=5 k \\
& \gamma_{R}=14 k
\end{aligned}
$$
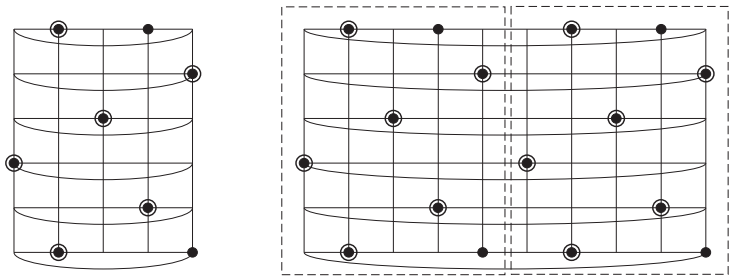

$n=5 k+1$

$\gamma_{R}=14 k+4$
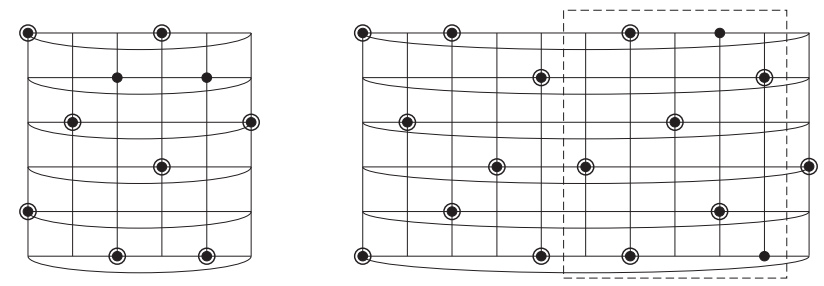

$$
\begin{aligned}
& n=5 k+2 \\
& \gamma_{R}=14 k+7
\end{aligned}
$$
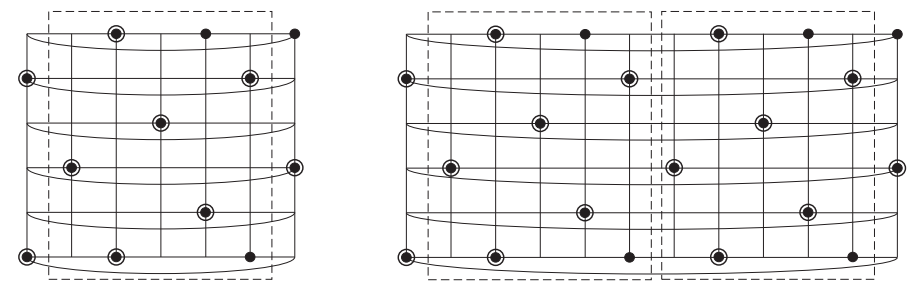

$$
\begin{aligned}
& n=5 k+3 \\
& \gamma_{R}=14 k+10
\end{aligned}
$$
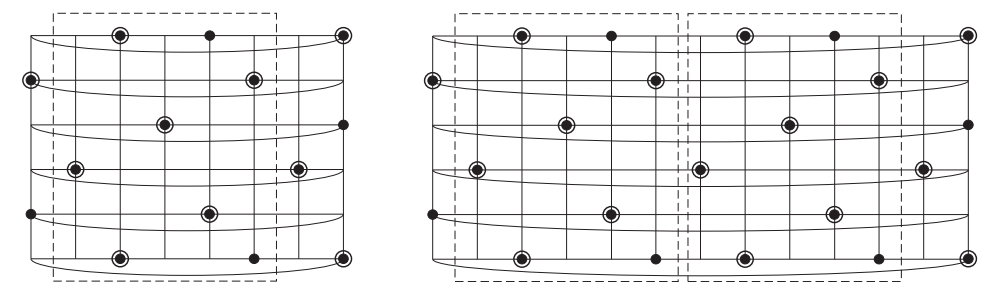

$$
\begin{aligned}
& n=5 k+4 \\
& \gamma_{R}=14 k+12
\end{aligned}
$$
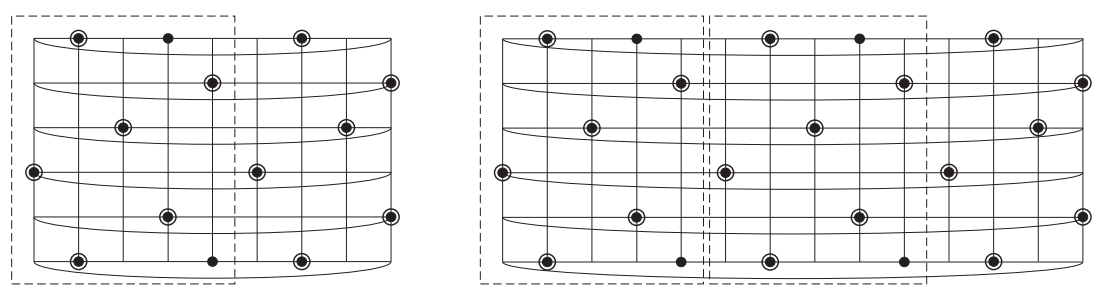

Figure 18: $C_{n} \square P_{6}$ 


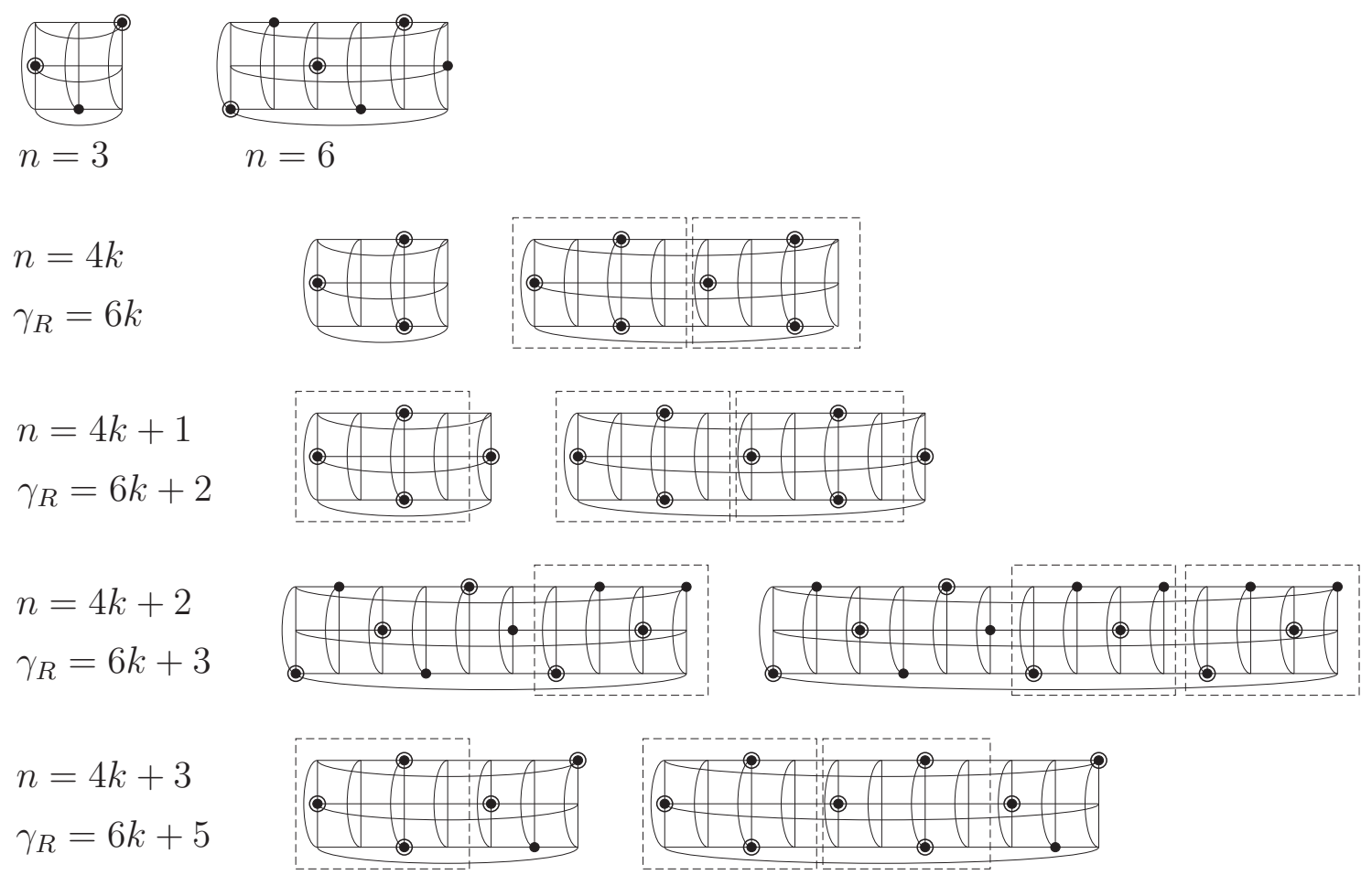

Figure 19: $C_{n} \square C_{3}$
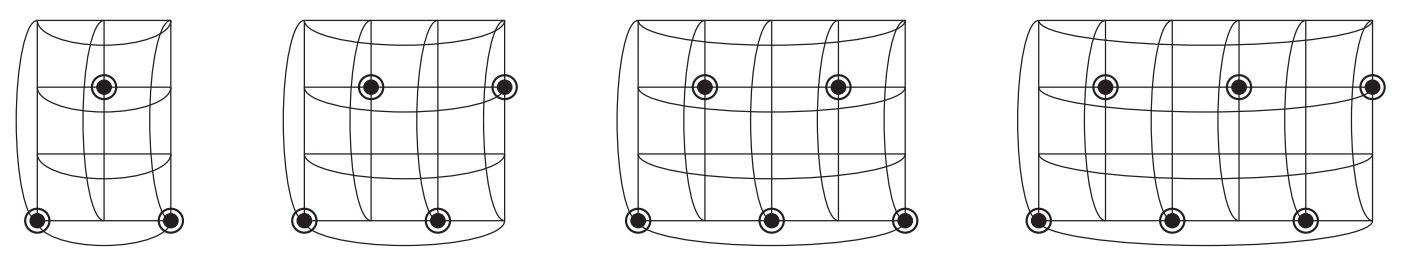

$$
n=3
$$$$
n=4
$$$$
n=5
$$$$
n=6
$$

Figure 20: $C_{n} \square C_{4}$ 


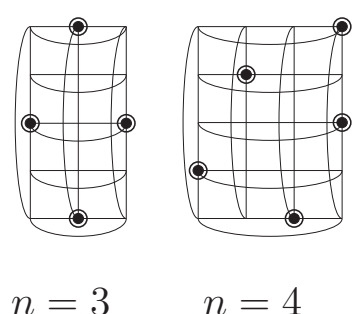

$$
\begin{gathered}
n=5 k \\
\gamma_{R}=10 k
\end{gathered}
$$
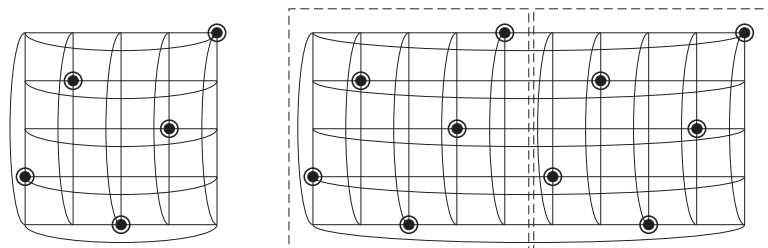

$$
\begin{aligned}
& n=5 k+1 \\
& \gamma_{R}=10 k+4
\end{aligned}
$$
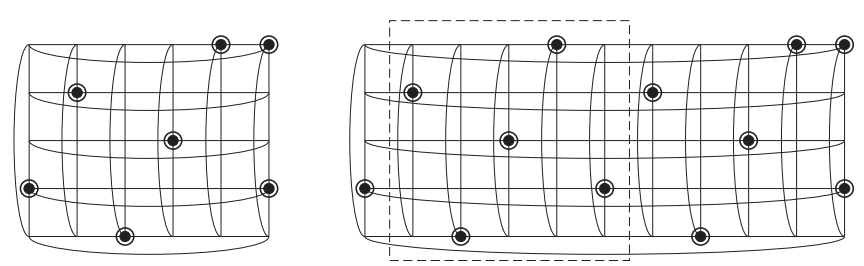

$$
\begin{aligned}
& n=5 k+2 \\
& \gamma_{R}=10 k+6
\end{aligned}
$$
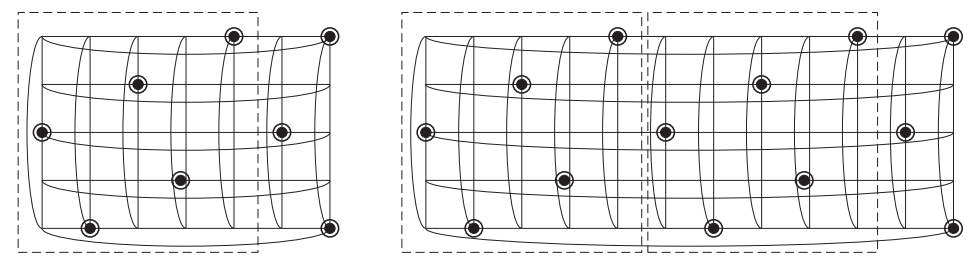

$$
\begin{aligned}
& n=5 k+3 \\
& \gamma_{R}=10 k+8
\end{aligned}
$$
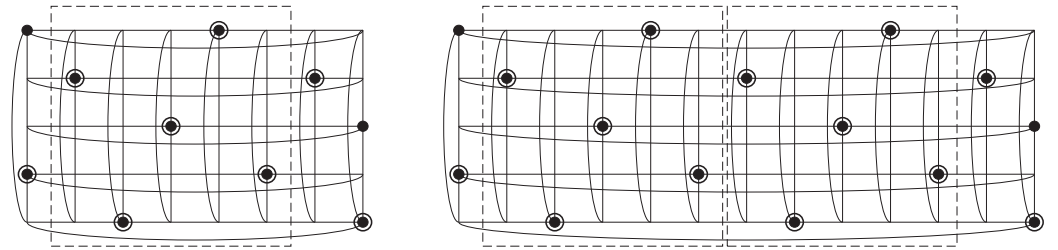

$$
\begin{aligned}
& n=5 k+4 \\
& \gamma_{R}=10 k+10
\end{aligned}
$$
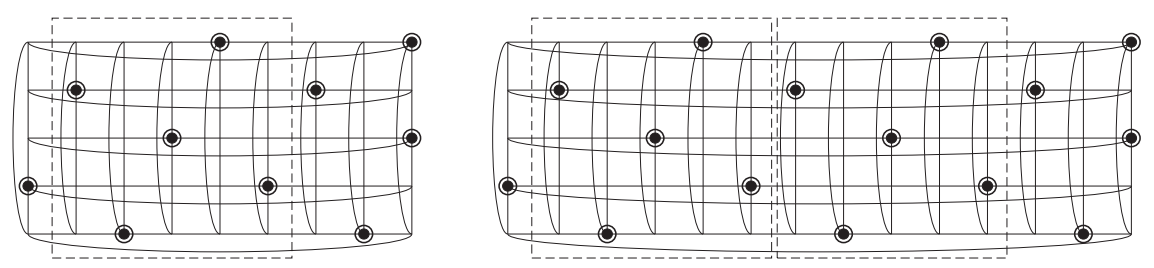

Figure 21: $C_{n} \square C_{5}$ 

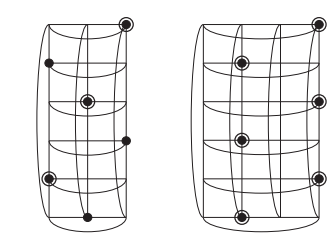

$n=3$

$n=4$

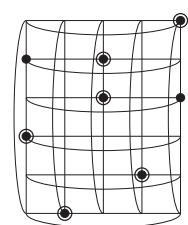

$n=5$

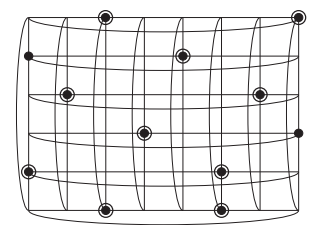

$n=8$

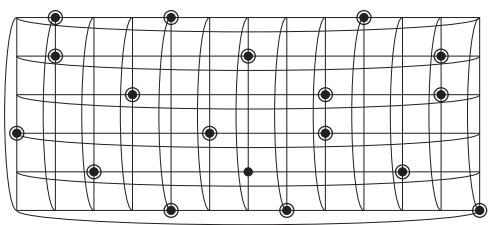

$n=13$

$n=6 k$

$\gamma_{R}=16 k$
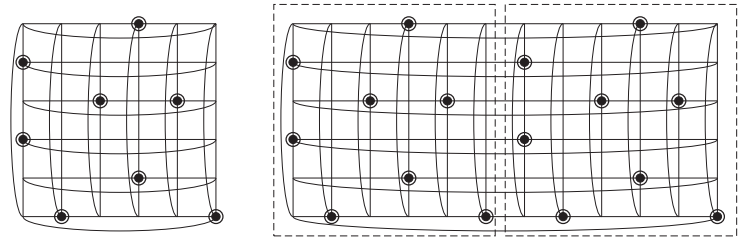

$n=6 k+4$

$\gamma_{R}=16 k+12$
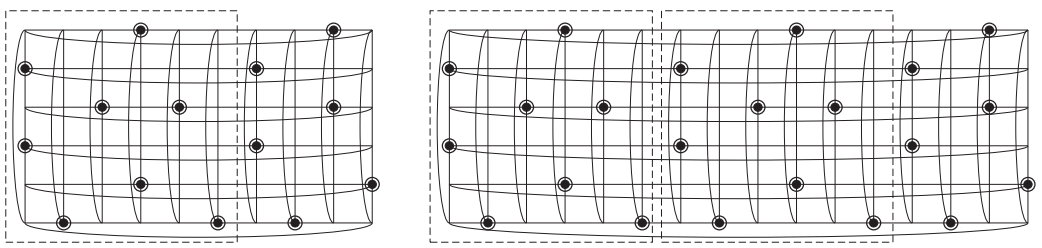

$n=6 k+5$

$\gamma_{R}=16 k+14$
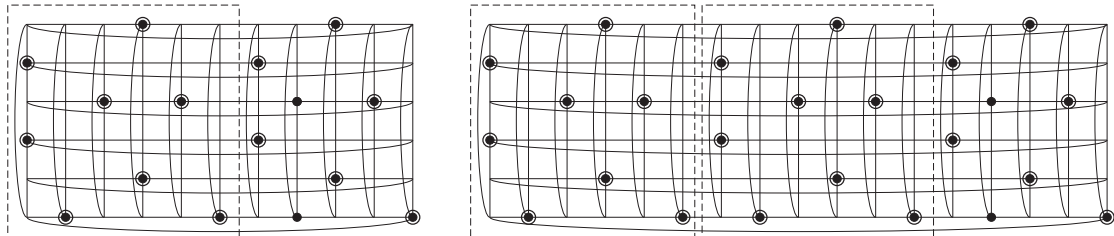

$n=18 k+1$

$\gamma_{R}=48 k+4$

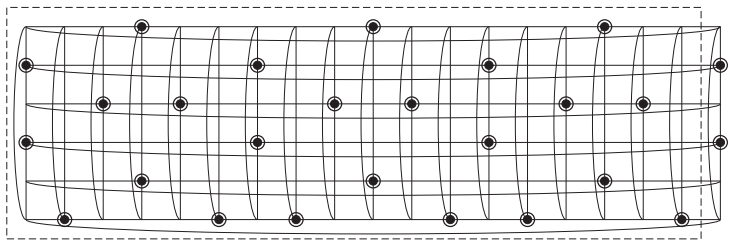

$n=18 k+7$

$\gamma_{R}=48 k+20$

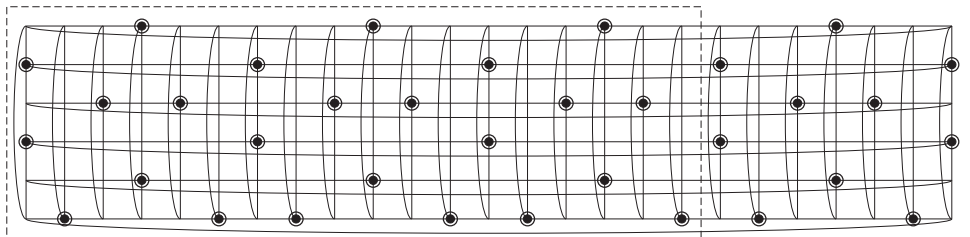

$n=18 k+13$

$\gamma_{R}=48 k+35$

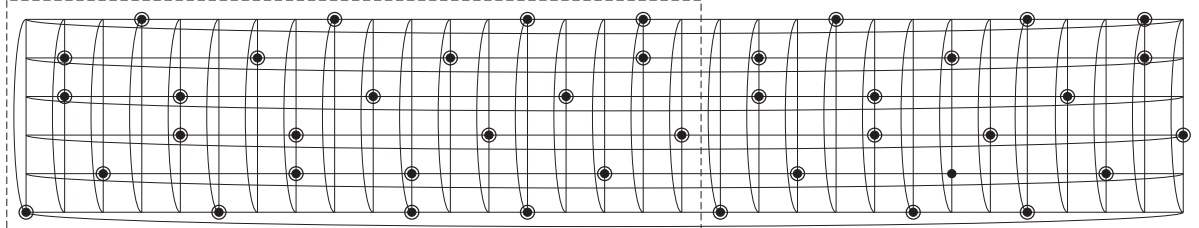

Figure 22: $C_{n} \square C_{6}$ 


$$
\begin{gathered}
n=18 k+2 \\
\gamma_{R}=48 k+7
\end{gathered}
$$

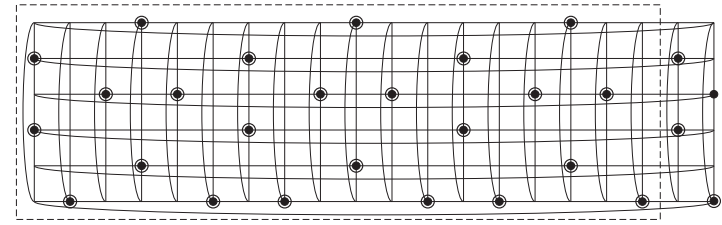

$$
\begin{aligned}
& n=18 k+8 \\
& \gamma_{R}=48 k+22
\end{aligned}
$$

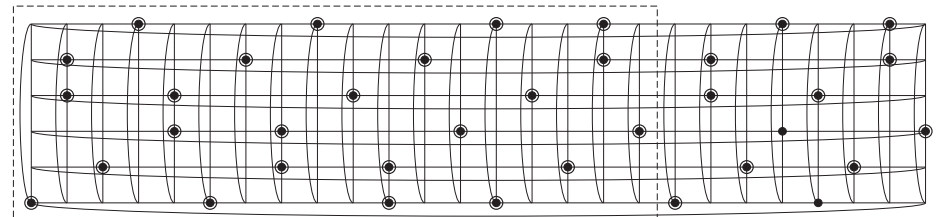

$$
\begin{aligned}
& n=18 k+14 \\
& \gamma_{R}=48 k+39
\end{aligned}
$$

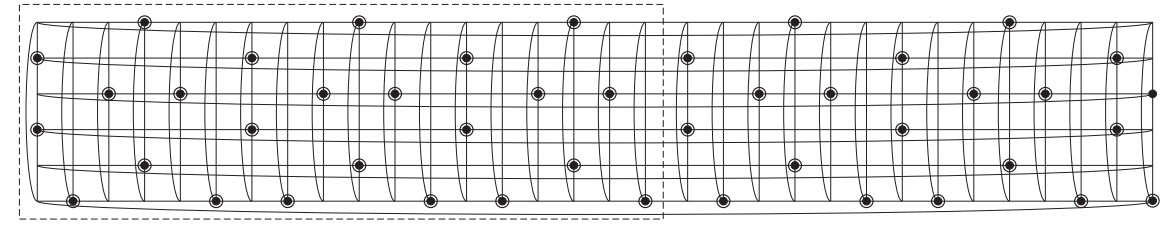

$$
\begin{aligned}
& n=18 k+3 \\
& \gamma_{R}=48 k+9
\end{aligned}
$$

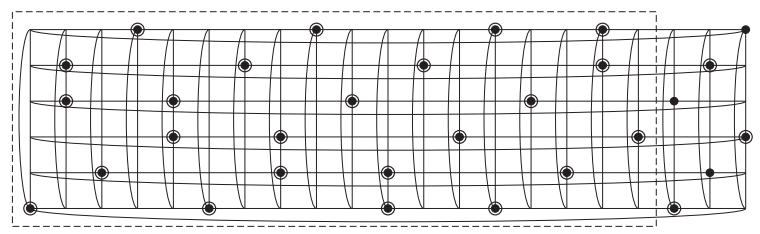

$$
\begin{aligned}
& n=18 k+9 \\
& \gamma_{R}=48 k+26
\end{aligned}
$$

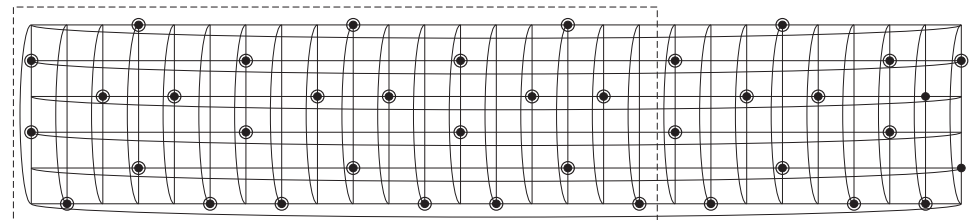

$$
\begin{aligned}
& n=18 k+15 \\
& \gamma_{R}=48 k+42
\end{aligned}
$$

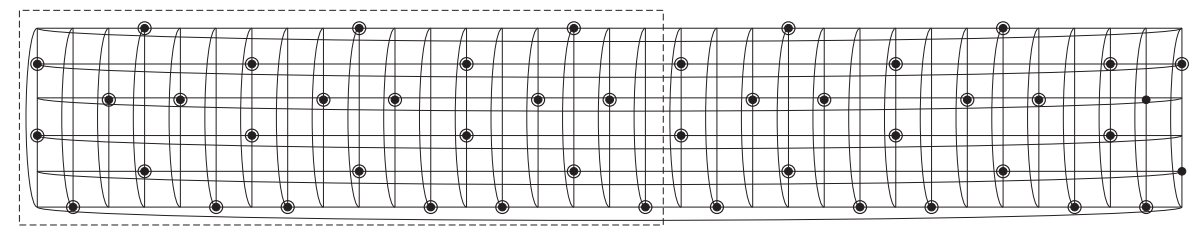

Figure 23: $C_{n} \square C_{6}$ 